November 1, 2018

hep-th/0411238

CERN-PH-TH/2004-222

HUTP-04/A0046

\title{
Toric Geometry, Sasaki-Einstein Manifolds and a New Infinite Class of AdS/CFT Duals
}

\author{
Dario Martelli ${ }^{1}$ and James Sparks ${ }^{2}$ \\ 1: Department of Physics, CERN Theory Division \\ 1211 Geneva 23, Switzerland \\ 2: Department of Mathematics, Harvard University \\ One Oxford Street, Cambridge, MA 02318, U.S.A. \\ and \\ Jefferson Physical Laboratory, Harvard University \\ Cambridge, MA 02138, U.S.A. \\ dario.martelli@cern.ch sparks@math.harvard.edu
}

\begin{abstract}
Recently an infinite family of explicit Sasaki-Einstein metrics $Y^{p, q}$ on $S^{2} \times S^{3}$ has been discovered, where $p$ and $q$ are two coprime positive integers, with $q<p$. These give rise to a corresponding family of Calabi-Yau cones, which moreover are toric. Aided by several recent results in toric geometry, we show that these are Kähler quotients $\mathbb{C}^{4} / / U(1)$, namely the vacua of gauged linear sigma models with charges $(p, p,-p+q,-p-q)$, thereby generalising the conifold, which is $p=1, q=0$. We present the corresponding toric diagrams and show that these may be embedded in the toric diagram for the orbifold $\mathbb{C}^{3} / \mathbb{Z}_{p+1} \times \mathbb{Z}_{p+1}$ for all $q<p$ with fixed $p$. We hence find that the $Y^{p, q}$ manifolds are AdS/CFT dual to an infinite class of $\mathcal{N}=1$ superconformal field theories arising as IR fixed points of toric quiver gauge theories with gauge group $S U(N)^{2 p}$. As a non-trivial example, we show that $Y^{2,1}$ is an explicit irregular Sasaki-Einstein metric on the horizon of the complex cone over the first del Pezzo surface. The dual quiver gauge theory has already been constructed for this case and hence we can predict the exact central charge of this theory at its IR fixed point using the AdS/CFT correspondence. The value we obtain is a quadratic irrational number and, remarkably, agrees with a recent purely field theoretic calculation using $a$-maximisation.
\end{abstract}




\section{Introduction and summary}

The AdS/CFT correspondence [1] predicts that type IIB string theory on $\operatorname{Ad} S_{5} \times$ $Y_{5}$, with appropriately chosen self-dual five-form flux, is dual to an $\mathcal{N}=1$ fourdimensional superconformal field theory whenever $Y_{5}$ is Sasaki-Einstein [2, 3, 4, 5]. This latter condition may be defined as saying that the metric cone over $Y_{5}$

$$
\mathrm{d} s^{2}\left(C\left(Y_{5}\right)\right)=\mathrm{d} r^{2}+r^{2} \mathrm{~d} s^{2}\left(Y_{5}\right)
$$

is Ricci-flat Kähler i.e. Calabi-Yau. The superconformal field theory may be thought of as arising from a stack of D3-branes sitting at the tip of the Calabi-Yau cone. Notice that, unless $Y_{5}$ is the round metric on $S^{5}$, appropriately normalised, the tip of the cone at $r=0$ will be singular.

It is a striking fact that, until very recently, the only Sasaki-Einstein five-manifolds that were known explicitly in the literature ${ }^{1}$ were precisely the round metric on $S^{5}$ and the homogeneous metric $T^{1,1}$ on $S^{2} \times S^{3}$, or quotients thereof. For the fivesphere the Calabi-Yau cone is simply $\mathbb{C}^{3}$ and the dual superconformal field theory is the maximally supersymmetric $\mathcal{N}=4 S U(N)$ theory. For $T^{1,1}$ the Calabi-Yau cone is the conifold and the dual $\mathcal{N}=1$ superconformal field theory was given in [3, [5].

Due to the rather limited number of examples in the literature detailed tests of the AdS/CFT conjecture for more interesting geometries have been lacking ${ }^{2}$. Indeed, one is restricted to quotients (orbifolds) of $S^{5}$ and $T^{1,1}$. These have been extensively studied using orbifold techniques which by now are completely standard. For example, Klebanov and Witten argued that the field theory for $T^{1,1}$ may be obtained via a relevant deformation of the $\mathcal{N}=2$ orbifold $S^{5} / \mathbb{Z}_{2}$.

However, this has changed drastically with the recent discovery [6] of a countably infinite class of explicit Sasaki-Einstein metrics on $Y^{p, q} \cong S^{2} \times S^{3}$. These were initially found by reduction and T-duality of a class of supersymmetric M-theory solutions discovered in [7]. The family is characterised by two relatively prime positive integers $p, q$, with $q<p$. A particularly interesting feature of these Sasaki-Einstein manifolds is that there are countably infinite classes which are both quasi-regular and irregular.

\footnotetext{
${ }^{1}$ E. Calabi has constructed an explicit Kähler-Einstein metric on del Pezzo 6 - recall that this is the blow-up of $\mathbb{C P}^{2}$ at 6 points - with a certain symmetric configuration of the 6 blown-up points. The corresponding Sasaki-Einstein metric on $\# 6\left(S^{2} \times S^{3}\right)$ is thus also explicit. This metric has apparently never been published. We thank S.-T. Yau for pointing this out to us.

${ }^{2}$ Although one can still deduce some geometric information for the regular Sasaki-Einstein manifolds $\# l\left(S^{2} \times S^{3}\right)$, which are $U(1)$ bundles over del Pezzo surfaces with $l$ points blown up, $l=3, \ldots, 8$, even though the general metrics are not known explicitly.
} 
These terms are not to be confused with regularity of the metric: the metrics are all smooth metrics on $S^{2} \times S^{3}$. Rather, they refer to properties of the orbits of a certain Killing vector field. Indeed, on any Sasaki-Einstein manifold $Y$ there exists a canonically defined Killing vector field $K$, called the Reeb vector in the mathematics literature. The orbits of this Killing vector field may or may not close. If they close then there is a (locally free) $U(1)$ action on $Y$ and such Sasaki-Einstein manifolds are called quasi-regular. The geometries $Y^{p, q}$ with $4 p^{2}-3 q^{2}$ a square are examples of such manifolds. If the orbits of the Reeb vector field do not close the Killing vector generates an action of $\mathbb{R}$ on $Y$, with the orbits densely filling the orbits of a torus, and the Sasaki-Einstein manifold is said to be irregular. The geometries $Y^{p, q}$ with $4 p^{2}-3 q^{2}$ not a square are the first examples of such geometries in the literature ${ }^{3}$. Another interesting feature of these metrics is that the volumes are always given by a quadratic irrational number times the volume of the round metric on $S^{5}$ - recall a quadratic irrational is of the form $a+b \sqrt{c}$ where $a, b \in \mathbb{Q}, c \in \mathbb{N}$. Moreover, the volumes are rationally related to that of $S^{5}$ if and only if the Sasaki-Einstein is quasi-regular.

Recall that all four-dimensional $\mathcal{N}=1$ superconformal field theories possess an $\mathrm{R}$-symmetry, commonly referred to as the $U(1) \mathrm{R}$-symmetry. However, crucially this symmetry is not always a $U(1)$ symmetry - this is true only if the R-charges of all the fields are rational. In general, this is not true, as exemplified by the recent work of [9]. In the latter reference it is shown that the exact R-symmetry of a superconformal field theory maximises a certain combination of 't Hooft anomalies $a_{\text {trial }}(R)=\left(9 \operatorname{Tr} R^{3}-3 \operatorname{Tr} R\right) / 32$. The maximal value is then precisely the exact $a$ central charge of the superconformal field theory. Since one is maximising a cubic with rational coefficients, the resulting $\mathrm{R}$-charges are always algebraic numbers. Recall that in AdS/CFT the R-symmetry is precisely dual to the canonical Killing vector field $K$ discussed above. Moreover, the central charge $a_{Y}$ for the field theory dual to $Y$ is inversely proportional to its volume. In particular, we have [10]

$$
\frac{a_{Y}}{a_{S^{5}}}=\frac{\operatorname{vol}\left(S^{5}\right)}{\operatorname{vol}(Y)} .
$$

It is thus clearly of interest to identify the dual superconformal field theories for the Sasaki-Einstein manifolds $Y^{p, q}$, so as to compare the exact results on both sides of the duality. In this paper we take the first substantial steps in this program by analysing

\footnotetext{
${ }^{3}$ Thus disproving a conjecture of Cheeger and Tian 8 that such examples do not exist. We thank the referee for drawing our attention to this reference.
} 
in considerable detail the geometry of the manifolds $Y^{p, q}$, and the associated CalabiYau cones. The results allow us to show that the metrics $Y^{p, q}$ are dual to a class of $\mathcal{N}=1$ superconformal field theories arising as IR fixed points of certain toric quiver gauge theories, with gauge group $S U(N)^{2 p}$.

The case $p=2, q=1$ is somewhat special. This corresponds to the geometry with largest volume, and is an irregular metric. The dual field theory therefore has the smallest central charge within the family, and moreover is expected to be quadratic irrational. Rather surprisingly, we find that the metric $Y^{2,1}$ turns out to be an explicit metric on the horizon of the complex cone over the first del Pezzo surface. For this, the corresponding $S U(N)^{4}$ quiver gauge theory and superpotential have already been identified [11. We can then compute the central charge (1.2) and also the Rcharges of the baryons for this theory using AdS/CFT, where the baryons correspond to D3-branes wrapped over 3-cycles whose metric cones are supersymmetric cycles (complex divisors) in the cone over $Y^{2,1}$. The values we find are all quadratic irrational numbers. At first sight these results present a puzzle, as the central charge computed in [9, 12, 13] was found to be a rational number. However, a closer inspection of the quiver theory shows that the $a$-maximisation calculation is somewhat more subtle in this case ${ }^{4}$. Indeed, using $a$-maximisation 9] applied to the quiver theory, the authors of [14] find a central charge, as well as R-charges, which agree perfectly with the values obtained using the geometrical results of this paper. This constitutes an extremely beautiful test of the AdS/CFT correspondence, as well as the general $a-$ maximisation procedure advocated in 9 .

Given the results presented here, in principle the duals to the remaining geometries, with general $p$ and $q, q<p$, can be constructed using the "toric algorithm" of [11]. These will provide an infinite series of $\mathcal{N}=1$ superconformal field theories, whose central charges are generically quadratic irrational. It will be interesting to obtain these explicitly, and to compare the results of $a$-maximisation for these theories with the various geometrical results presented in this paper. However, we leave these calculations for future work.

As a final point, we note that in [15] a generalisation of the metrics $Y^{p, q}$ to all dimensions was presented (see also references [16] and [17] for a generalisation of this generalisation). In particular there are countably infinite classes of supersymmetric solutions $A d S_{4} \times Y_{7}$ to M-theory, which will have three-dimensional CFT duals, where

\footnotetext{
${ }^{4}$ We are very grateful to M. Bertolini, F. Bigazzi, A. Hanany, K. Intriligator, and B. Wecht for discussions on this issue.
} 
the metric $Y_{7}$ is built using any positive curvature Kähler-Einstein metric in real dimension four [15]. These have been classified [18, 19]. For the case when the KählerEinstein manifold is toric, one has only three cases: $\mathbb{C P}^{2}, \mathbb{C P}^{1} \times \mathbb{C P}^{1}$, and $d P_{3}$, where the latter is the third del Pezzo surface. Using the techniques developed in this paper, one can show that for the first two cases the metric cones over $Y_{7}$ are given by Kähler quotients $\mathbb{C}^{5} / / U(1)$, and $\mathbb{C}^{6} / / U(1)^{2}$, respectively, where the various $U(1)$ charges are, with appropriate definitions ${ }^{5}$ of the Chern numbers $p$ and $k, Q=(p, p, p,-3 p+k,-k)$ and $Q_{1}=(p, p, 0,0,-2 p+k,-k), Q_{2}=(0,0, p, p,-2 p+k,-k)$, respectively.

\section{Outline}

The first point to note about the manifolds $Y^{p, q}$, and their associated Calabi-Yau cones, is that they are all toric. This essentially means that there is an effective action of a torus $\mathbb{T}^{3} \cong U(1)^{3}$ on $C\left(Y^{p, q}\right)$ which preserves the symplectic form of the cone and commutes with the homothetic $\mathbb{R}^{+}$action. Indeed, this torus action is an isometry, and so also preserves the metric. The torus action and symplectic form then allow us to define a moment map, $\mu: C\left(Y^{p, q}\right) \rightarrow \mathbb{R}^{3}$. The image in $\mathbb{R}^{3}$ is always a good convex rational polyhedral cone in $\mathbb{R}^{3}[20$. These terms will be explained more carefully later. However, roughly this is a convex cone formed by intersecting some number of planes through the origin. The moment map exhibits $C\left(Y^{p, q}\right)$ as a $\mathbb{T}^{3}$ fibration over this moment cone, with the fibres collapsing over the faces, or facets, of the cone in a way determined by the normal vectors to the facets. We shall find explicitly that the moment cone for $Y^{p, q}$ is a four-faceted good strictly convex rational polyhedral cone.

Having computed the moment cone for $C\left(Y^{p, q}\right)$ we may then apply a Delzant theorem 21] for symplectic toric cones worked out recently in [20. In physics terms, this takes the combinatorial data defining the moment cone and uses it to produce a gauged linear sigma model [22]. By construction the classical vacuum of the linear sigma model is precisely the Calabi-Yau cone one started with. More mathematically, this would be called a symplectic - or, more precisely, Kähler - quotient of $\mathbb{C}^{d}$ by a compact abelian group. The final result is:

- The metric cones over $Y^{p, q}$ are explicit Calabi-Yau metrics for the $U(1)$ gauged linear sigma model on $\mathbb{C}^{4}$ with charges $(p, p,-p+q,-p-q)$, and zero FayetIliopoulos parameter.

\footnotetext{
${ }^{5}$ In particular, the definitions here are different from those in [15].
} 
If we denote the vacuum of a linear sigma model by $X=\mathbb{C}^{4} / / U(1)$, then it is easy to see that, rather generally, $c_{1}(X)=0$ is equivalent to the charges of the $U(1)$ gauge group summing to zero. Clearly this is true for the gauged linear sigma model above, and hence $X$ is indeed topologically Calabi-Yau. In this process we lose precise information about the metric - in particular, the induced metric from $\mathbb{C}^{4}$ is not Ricci-flat. However, we have now gained an explicit description of the CalabiYau singularity. Indeed, by constructing invariant monomials one also obtains an algebraic description of the singularity.

We may then give the toric diagram for the Calabi-Yau singularity. This may be realised as an integral polytope in $\mathbb{R}^{2}$. Roughly, the four outward pointing primitive normal vectors that define the moment cone lie in a plane as a result of the CalabiYau condition. Projecting these vectors onto this plane yields the vertices of the toric diagram for a minimal presentation of the singularity. We show that the resulting toric diagrams may all be embedded inside that of the orbifold $\mathbb{C}^{3} / \mathbb{Z}_{p+1} \times \mathbb{Z}_{p+1}$ where the two factors are generated by $\left(\omega_{p+1}, \omega_{p+1}^{-1}, 1\right),\left(\omega_{p+1}, 1, \omega_{p+1}^{-1}\right) \subset S U(3)$, respectively, where $\omega_{p+1}$ is a $(p+1)$-th root of unity. The vertices of the polytope are then $(0,0)$, $(0, p+1)$ and $(p+1,0)$ (the position of the origin is irrelevant) and we show that the toric diagram for $C\left(Y^{p, q}\right)$ lives inside this polytope for all $q<p$ and fixed $p$. Geometrically, this means that the Calabi-Yau cone $C\left(Y^{p, q}\right)$ may be obtained by (partial) toric crepant resolution of the orbifold [5, 23].

Also, as part of our general analysis, we find a class of supersymmetric submanifolds in the geometries $C\left(Y^{p, q}\right)$. Specifically, we show that the cones over the special orbits of the cohomogeneity one action on $Y^{p, q}$ are calibrated submanifolds - in fact complex divisors - of the Calabi-Yau. Recall that D3-branes wrapped over the horizon 3cycles are dual to baryons in the AdS/CFT correspondence [24, 25]. We compute the volumes of these submanifolds, and hence give a prediction for the $\mathrm{R}$-charges of the corresponding baryons.

Given the toric diagram for $C\left(Y^{p, q}\right)$ there are methods to construct a superconformal field theory, whose Higgs branch is the toric variety $X \cong C\left(Y^{p, q}\right)$, purely from the combinatorial data that defines $X$ [11]. Indeed, the point is that the field theory for the orbifold $\mathbb{C}^{3} / \mathbb{Z}_{p+1} \times \mathbb{Z}_{p+1}$, in which the geometries are "embedded", is known from standard orbifold techniques. The Calabi-Yau cones $C\left(Y^{p, q}\right)$ are obtained by partial resolution, which amounts to turning on specific combinations of Fayet-Iliopoulos parameters in the gauged linear sigma model. The field theories in question are then rather conventional quiver gauge theories with polynomial super- 
potentials. The number of nodes of the quiver is simply twice the area of the toric diagram, which is $2 p$ for all $q$ with fixed $p$.

Rather surprisingly, we find that the toric diagram for $Y^{2,1}$ is precisely the same as that for the complex cone over the first del Pezzo surface. Recall that the latter is the blow-up of $\mathbb{C P}^{2}$ at one point, and that the complex cone over this is indeed a real cone over $S^{2} \times S^{3}$. It follows that $Y^{2,1}$, which is irregular, is an explicit SasakiEinstein metric on the horizon, or boundary, of this cone. This is interesting, since the higher del Pezzo surfaces, which are $\mathbb{C P}^{2}$ with $3 \leq r \leq 8$ generic points blown up, admit Kähler-Einstein metrics [18, 19]. The complex cones then carry regular SasakiEinstein metrics. The case of one or two points blown up has always been something of a puzzle, since these del Pezzos do not admit Kähler-Einstein metrics and thus the Sasaki-Einstein metrics associated to the complex cones could not possibly be regular. We have thus resolved this puzzle, at least in the case of one blow-up.

The quiver gauge theory dual to the complex cone over the first del Pezzo surface has been presented in the literature [1]. The AdS/CFT correspondence then predicts the exact central charge of this theory in the IR. Using the explicit metric $Y^{2,1}$, the result we obtain is

$$
\frac{a_{S^{5}}}{a_{Y^{2,1}}}=\frac{\operatorname{vol}\left(Y^{2,1}\right)}{\operatorname{vol}\left(S^{5}\right)}=\frac{13 \sqrt{13}+46}{12 \cdot 27} \sim \frac{7.74}{27} .
$$

Remarkably, this value coincides precisely with a recent application of $a$-maximisation 9] to the quiver gauge theory [14. Moreover, we also find perfect agreement for the charges of $\left(S U(2)_{F}\right.$ singlet) baryons in the gauge theory.

The plan of the rest of the paper is as follows. In Section 2, after recalling some basic facts about Sasaki-Einstein geometry, we give a summary of the construction of the metrics $Y^{p, q}$, and recall several of their features. Section 3 contains a review of symplectic toric geometry - in particular toric contact geometry - which we use extensively in the remainder of the paper. In Section 4 we compute the image of the moment map associated to the toric Calabi-Yau cones $C\left(Y^{p, q}\right)$. In section 5 we apply a Delzant construction to obtain a gauged liner sigma model (GLSM) description of the Calabi-Yau spaces. Moreover we analyse directly the structure of the moduli space of vacua of the GLSM in Section 5.3. In Section 6 the associated toric Gorenstein singularities are described. In Section 7 we demonstrate that $Y^{2,1}$ is an irregular metric on the horizon of the complex cone over the first del Pezzo surface, and exhibit an explicit (non-Kähler and non-Einstein) metric on the latter. Section 8 
concludes with a comparison of the geometrical results obtained here with the results of $a$-maximisation applied to the quiver gauge theory corresponding to the complex cone over the first del Pezzo surface [14]. In Appendix A the techniques used in the paper, which perhaps are unfamiliar to many physicists, are applied to the familiar example of the conifold.

\section{Sasaki-Einstein Metrics on $S^{2} \times S^{3}$}

In this section we review the geometry of the recently discovered Sasaki-Einstein metrics on $S^{2} \times S^{3}[6]$. There is an infinite family of such metrics, labeled by two coprime integers $p>1, q<p$ - we refer to these as $Y^{p, q}$. Geometrically they are all $U(1)$ principle bundles ${ }^{6}$ over an axially squashed $S^{2}$ bundle over a round $S^{2}$. The integers label the twisting, or Chern numbers, of the $U(1)$ bundle over the two twocycles, with the constraint $q<p$ arising as a regularity condition on the metric. The manifolds are all cohomogeneity one. The fact that they are all topologically $S^{2} \times S^{3}$ follows from a theorem of Smale [26] on the classification of five-manifold topology.

In the following we first recall basic material about Sasakian-Einstein geometry and then turn to the metrics $Y^{p, q}$.

\subsection{Sasakian-Einstein geometry}

A Sasaki-Einstein manifold may be defined as a complete positive curvature Einstein manifold $^{7}$ whose metric cone is Ricci-flat Kähler i.e. a Calabi-Yau cone. The structure of a Sasaki-Einstein manifold may thus be thought of as "descending" from the Calabi-Yau structure of its metric cone (1.1). In particular, contracting the Euler vector $r \partial / \partial r$, which generates the homothetic $\mathbb{R}^{+}$action on the cone, into the Kähler form gives rise to a one-form on the base of the cone, $Y$. The dual of this is a constant norm Killing vector field - called the Reeb vector in the mathematical literature - which via the AdS/CFT correspondence is isomorphic to the R-symmetry of the dual field theory. The Killing vector defines a foliation of the Sasaki-Einstein manifold, and one finds that the transverse leaves have a Kähler-Einstein structure.

\footnotetext{
${ }^{6}$ This $U(1)$ is not to be confused with the isometry generated by the Reeb vector. The latter is embedded non-trivially inside the torus defined by this $U(1)$ and and $U(1)$ that rotates the axially squashed $S^{2}$ fibre.

${ }^{7}$ We also require simply-connectedness. This is not strictly necessary. However, given this condition we can use a theorem which relates contact structures to the existence of globally-defined Killing spinors. The latter is the physical property that we wish our manifolds to possess.
} 
More precisely, one can write the local form of the metric as follows:

$$
\mathrm{d} s^{2}(Y)=\mathrm{d} s_{4}^{2}+\left(\frac{1}{3} \mathrm{~d} \psi^{\prime}+\sigma\right)^{2}
$$

where $\mathrm{d} s_{4}^{2}$ is a local Kähler-Einstein metric. In particular we have that

$$
\begin{aligned}
\mathrm{d} \sigma & =2 J_{4} \\
\mathrm{~d} \Omega_{4} & =i 3 \sigma \wedge \Omega_{4}
\end{aligned}
$$

where $J_{4}$ and $\Omega_{4}$ are the local Kähler and holomorphic $(2,0)$ form for $\mathrm{d} s_{4}^{2}$, respectively. The Reeb Killing vector is given by

$$
K \equiv 3 \frac{\partial}{\partial \psi^{\prime}} .
$$

Sasaki-Einstein manifolds may then be classified into three families, according to the global properties of the orbits of this Killing vector field:

- If the orbits close, and moreover the associated $U(1)$ action is free, the SasakiEinstein manifold is said to be regular. The length of the orbits are then all equal. One thus has a principle $U(1)$ bundle over a four-dimensional base Kähler-Einstein manifold.

- Suppose that the isotropy group $\Gamma_{x}$ of at least one point $x$ is non-trivial. Notice that $\Gamma_{x}$ is necessarily isomorphic to $\mathbb{Z}_{m}$, for some integer $m$, since these are precisely the proper subgroups of $U(1)$. The $U(1)$ action is then locally free, meaning that the isotropy groups are all finite - note that the Killing vector cannot vanish anywhere since it has constant norm. The Sasaki-Einstein manifold is then said to be quasi-regular. In this case notice that the length of the orbit through $x$ is $1 / m$ times the length of the generic orbit. The quotient of any manifold by a locally free compact Lie group action is canonically an orbifold. One thus has a principle orbifold $U(1)$ bundle, or orbibundle, over a KählerEinstein base orbifold. Moreover, the point $x$ will descend to a $\mathbb{Z}_{m}$-orbifold point $x$ in this base space.

- If the orbits do not close, the Sasaki-Einstein manifold is said to be irregular. In this case one does not have a well-defined quotient space. Note that such a Sasaki-Einstein manifold necessarily has at least a $U(1)^{d}$ isometry group, $d \geq 2$, with the orbits of the Killing vector filling out a dense subset of the 
orbits of the torus action. Indeed, the isometry group of a compact Riemannian manifold is always a compact Lie group. Hence the orbits of a Killing vector field define a one-parameter subgroup, the closure of which will always be an abelian subgroup and thus a torus. The dimension of the closure of the orbits is called the rank. Thus irregular Sasaki-Einstein manifolds have rank greater than 1.

The five-dimensional regular Sasaki-Einstein manifolds are classified completely [27. This follows since the smooth four-dimensional Kähler-Einstein metrics with positive curvature on the base have been classified by Tian and Yau [18, 19]. These include the special cases $\mathbb{C P}^{2}$ and $S^{2} \times S^{2}$, with corresponding Sasaki-Einstein manifolds being the homogeneous manifolds $S^{5}$ (or $S^{5} / \mathbb{Z}_{3}$ ) and $T^{1,1}$ (or $T^{1,1} / \mathbb{Z}_{2}$ ), respectively. For the remaining metrics, the base is a del Pezzo surface obtained by blowing up $\mathbb{C P}^{2}$ at $k$ generic points with $3 \leq k \leq 8$ and, although proven to exist, the generic metrics are not known explicitly.

We emphasise the lack of existence of Kähler-Einstein metrics on the del Pezzo surfaces with one or two points blown up, as this will play an important role later. This fact is actually rather simple to understand. It is a fairly straightforward calculation [28] to show that the Lie algebra $\mathrm{H}$ generated by holomorphic vector fields on a Kähler-Einstein manifold is a complexification of the Lie algebra generated by Killing vector fields i.e. isometries. The latter is always a reductive algebra (meaning it is the sum of its centre together with a semi-simple algebra) but for the first and second del Pezzo surfaces the algebra $\mathrm{H}$ is not reductive. Clearly then $\mathrm{H}$ being reductive is always necessary. This is Matsushima's Theorem [28]. One also requires that the anti-canonical bundle be ample, that is $c_{1}>0$, otherwise the putative Kähler-Einstein metric would be indefinite. In complex dimension two, these necessary conditions are in fact sufficient for existence of a Kähler-Einstein metric [18, 19], and this leads to the list stated above.

It was only recently [29, 30, 31, 32] that quasi-regular Sasaki-Einstein metrics were shown to exist on $\# l\left(S^{2} \times S^{3}\right)$ with $l=1, \ldots, 9$. In particular, there are 14 known inhomogeneous Sasaki-Einstein metrics on $S^{2} \times S^{3}$. We stress that the proof of this is via existence arguments, rather than giving explicit metrics. Specifically, one uses a modification of Yau's argument to prove existence of Kähler-Einstein metrics on certain complex orbifolds, and then builds the appropriate $U(1)$ orbibundle over these to obtain Sasaki-Einstein manifolds. One can also obtain quasi-regular geometries 
rather trivially by taking quotients of the explicit regular geometries discussed above by appropriate freely-acting finite groups. For example, one can take a freely-acting finite subgroup of $S U(3)$ and quotient $S^{5} \subset \mathbb{C}^{3}$ by the induced action.

\subsection{The metrics $Y^{p, q}$}

We will now review, as well as work out some new, properties of the Sasaki-Einstein metrics $Y^{p, q}$ on $S^{2} \times S^{3}$. These were presented in [6] in the following local form:

$$
\begin{aligned}
\mathrm{d} s^{2} & =\frac{1-c y}{6}\left(\mathrm{~d} \theta^{2}+\sin ^{2} \theta \mathrm{d} \phi^{2}\right)+\frac{1}{w(y) q(y)} \mathrm{d} y^{2}+\frac{q(y)}{9}(\mathrm{~d} \psi-\cos \theta \mathrm{d} \phi)^{2} \\
& +w(y)[\mathrm{d} \alpha+f(y)(\mathrm{d} \psi-\cos \theta \mathrm{d} \phi)]^{2} \\
& \equiv \mathrm{d} s^{2}(B)+w(y)[\mathrm{d} \alpha+A]^{2}
\end{aligned}
$$

where

$$
\begin{aligned}
w(y) & =\frac{2\left(a-y^{2}\right)}{1-c y} \\
q(y) & =\frac{a-3 y^{2}+2 c y^{3}}{a-y^{2}} \\
f(y) & =\frac{a c-2 y+y^{2} c}{6\left(a-y^{2}\right)} .
\end{aligned}
$$

For $c=0$ the metric takes the local form of the standard homogeneous metric on $T^{1,1}$. Otherwise, $c$ can be scaled to 1 by a diffeomorphism. Henceforth we assume this is the case.

\section{The base $B$}

The analysis of [6] first showed that the four dimensional space $B$ can be made into a smooth complete compact manifold with appropriate choices for the ranges of the coordinates. In particular, for ${ }^{8}$

$$
0<a<1
$$

one can take the ranges of the coordinates $(\theta, \phi, y, \psi)$ to be $0 \leq \theta \leq \pi, 0 \leq \phi \leq 2 \pi$, $y_{1} \leq y \leq y_{2}, 0 \leq \psi \leq 2 \pi$ so that the "base space" $B$ is an axially squashed $S^{2}$ bundle over a round $S^{2}$. The latter is parametrised by $\theta$, $\phi$, with $\psi$ being an azimuthal

\footnotetext{
${ }^{8}$ In the limit $a \rightarrow 1$ the two positive roots become equal and $y=1$ is a double root. In the case $a=1$ the metric is locally that of the round metric on $S^{5}$.
} 
coordinate on the axially squashed $S^{2}$ fibre. This bundle is geometrically twisted, and may be thought of as the $S^{2}$ bundle over $S^{2}$ formed by taking the tangent bundle of the round two-sphere and adding a point at infinity to each fibre. Now, the inclusion map $U(1) \hookrightarrow S O(3)$ induces a map $\mathbb{Z} \cong \pi_{1}(U(1)) \rightarrow \pi_{1}(S O(3)) \cong \mathbb{Z}_{2}$ which is reduction modulo 2 . Here we are thinking of $U(1)$ as the group in which the transition functions of $T S^{2}$ take their values, and $S O(3)$ as the structure group of the associated oriented $S^{2}$ bundle over $S^{2}$. Since $T S^{2}$ has Chern number $2 \cong 0 \bmod$ 2 , it follows that the $S^{2}$ bundle is trivial and thus the manifold $B$ is topologically a product space, $B \cong S^{2} \times S^{2}$. The range of $y$ is fixed so that $1-y>0, a-y^{2}>0$, $w(y)>0, q(y) \geq 0$. Specifically, $y_{i}$ are two zeroes of $q(y)$, i.e. are two roots of the cubic

$$
\mathcal{Q}(y) \equiv a-3 y^{2}+2 y^{3}=0 .
$$

If $0<a<1$ there are three real roots, one negative $\left(y_{1}\right)$ and two positive, the smallest being $y_{2}$. The values $y=y_{1}, y_{2}$ then correspond to the south and north poles of the axially squashed $S^{2}$ fibre. One may check explicitly that the metric is smooth here with the above identifications of coordinates.

\section{The circle fibration}

It was shown in [6] that for a countably infinite number of values of $a$, with $0<a<1$, one can now choose the period of $\alpha$ so as to describe a principle $S^{1}$ bundle over $B$. This is true if and only if the periods of $\mathrm{d} A$ are rationally related. Thus one requires

$$
P_{1}=\ell p, \quad P_{2}=\ell q
$$

with the periods $P_{i}, i=1,2$, given by

$$
P_{i}=\frac{1}{2 \pi} \int_{C_{i}} \mathrm{~d} A
$$

where $C_{1}$ and $C_{2}$ give the standard basis for the homology group of two-cycles on $B \cong S^{2} \times S^{2}$. In this case, one may take

$$
0 \leq \alpha \leq 2 \pi \ell
$$

and the five-dimensional space is then the total space of an $S^{1}$ fibration over $B \cong$ $S^{2} \times S^{2}$, with Chern numbers $p$ and $q$ over the two two-cycles. An explicit calculation shows that

$$
\frac{P_{1}}{P_{2}}=\frac{3}{2\left(y_{2}-y_{1}\right)} .
$$


Moreover, the function $y_{2}(a)-y_{1}(a)$ is a monotonic increasing function of $a$, taking the range $0<y_{2}(a)-y_{1}(a)<3 / 2$ thus implying a countably infinite number of solutions with $0<q / p<1$. Furthermore, for any $p$ and $q$ coprime, the space $Y^{p, q}$ is topologically $S^{2} \times S^{3}$ - see [6]. This follows from a result of Smale on the classification of five-manifold topology.

\section{The volumes}

One finds that

$$
\ell=\frac{q}{3 q^{2}-2 p^{2}+p\left(4 p^{2}-3 q^{2}\right)^{1 / 2}}
$$

and the volume of $Y^{p, q}$ is given by

$$
\operatorname{vol}\left(Y^{p, q}\right)=\frac{q^{2}\left[2 p+\left(4 p^{2}-3 q^{2}\right)^{1 / 2}\right]}{3 p^{2}\left[3 q^{2}-2 p^{2}+p\left(4 p^{2}-3 q^{2}\right)^{1 / 2}\right]} \pi^{3}
$$

which is a quadratic irrational number times the volume $\pi^{3}$ of a unit round $S^{5}$. We note that at fixed $p$ the volume is a monotonic function of $q$, and is bounded by the following values

$$
\operatorname{vol}\left(T^{1,1} / \mathbb{Z}_{p}\right)>\operatorname{vol}\left(Y^{p, q}\right)>\operatorname{vol}\left(S^{5} / \mathbb{Z}_{2} \times \mathbb{Z}_{p}\right)
$$

The rational case, which is easily seen to correspond to quasi-regular manifolds, is described by $p, q \in \mathbb{N}$, hcf $(p, q)=1, q<p$, which are solutions to the quadratic diophantine

$$
4 p^{2}-3 q^{2}=n^{2}
$$

for some $n \in \mathbb{Z}$. The solutions to this were given in closed form in [6].

\section{The isometry group}

The isometry group of the metrics (2.4) is clearly locally $S U(2) \times U(1) \times U(1)$, and in particular there are three commuting Killing vectors $\partial / \partial \phi, \partial / \partial \psi$, and $\partial / \partial \gamma$. Here we have defined

$$
\alpha \equiv \ell \gamma
$$

so that the three generators have canonical period $2 \pi$. For us it will be important to note that the global form of the effectively acting isometry group depends on $p$ and $q$. In particular, for both $p$ and $q$ odd it is $S O(3) \times U(1)^{2}$ otherwise it is $U(2) \times U(1)$. 
This will be explained later in Section 4. Note that this is precisely analogous to the case of the Einstein manifolds known in the physics literature as $T^{p, q}$. For these the effectively acting isometry group is shown [33] to be $S O(3) \times S U(2)$ when one integer is even, and $S O(4) \cong(S U(2) \times S U(2)) / \mathbb{Z}_{2}$ when both are odd. The latter of course includes the case of $T^{1,1}[3]$.

\section{The local Kähler-Einstein structure}

Employing the change of coordinates $\alpha=-\beta / 6-\psi^{\prime} / 6, \psi=\psi^{\prime}$ one can [6] bring the metric (2.4) into the local Sasaki-Einstein form (2.1). In particular

$$
\begin{aligned}
\mathrm{d} s^{2} & =\frac{1-y}{6}\left(\mathrm{~d} \theta^{2}+\sin ^{2} \theta \mathrm{d} \phi^{2}\right)+\frac{\mathrm{d} y^{2}}{w(y) q(y)}+\frac{1}{36} w(y) q(y)(\mathrm{d} \beta+\cos \theta \mathrm{d} \phi)^{2} \\
& +\frac{1}{9}\left[\mathrm{~d} \psi^{\prime}-\cos \theta \mathrm{d} \phi+y(\mathrm{~d} \beta+\cos \theta \mathrm{d} \phi)\right]^{2}
\end{aligned}
$$

The corresponding $J_{4}$ and $\Omega_{4}$, satisfying (2.2), can be taken as

$$
\begin{aligned}
J_{4} & =\frac{1-y}{6} \sin \theta \mathrm{d} \theta \wedge \mathrm{d} \phi+\frac{1}{6} \mathrm{~d} y \wedge(\mathrm{d} \beta+\cos \theta \mathrm{d} \phi) \\
\Omega_{4} & =\sqrt{\frac{1-y}{6 w(y) q(y)}}(\mathrm{d} \theta+i \sin \theta \mathrm{d} \phi) \wedge\left[\mathrm{d} y+i \frac{w(y) q(y)}{6}(\mathrm{~d} \beta+\cos \theta \mathrm{d} \phi)\right]
\end{aligned}
$$

while the Reeb Killing vector is given by

$$
K=3 \frac{\partial}{\partial \psi}-\frac{1}{2 \ell} \frac{\partial}{\partial \gamma} .
$$

Note that this has compact orbits when $\ell$ is a rational number and corresponds to the quasi-regular class, by definition. This is true if and only if (2.15) holds. If $\ell$ is irrational the generic orbits do not close, but instead densely fill the orbits of the torus generated by $[\partial / \partial \psi, \partial / \partial \gamma]$ and we thus fall into the irregular class. The rank of these metrics is thus equal to 2 . Note that the orbits close only over the submanifolds given by $y=y_{1}, y_{2}$. These are precisely the special ${ }^{9}$ orbits of the cohomogeneity one action.

\section{The Killing spinors}

To show that these manifolds admit globally defined Killing spinors one appeals to the following theorem [34]: every simply-connected spin Sasaki-Einstein manifold,

\footnotetext{
${ }^{9}$ The manifolds $Y^{p, q}$ are cohomogeneity one, meaning that the generic orbit under the action of the isometry group is codimension one. There are then always precisely two special orbits of higher codimension.
} 
where the latter is defined in terms of the existence of a certain contact structure, admits a solution to the Killing spinor equation. In particular we note that the dual one-form to $K$ is given by

$$
\eta=-2 y(\mathrm{~d} \alpha+A)+\frac{1}{3} q(y)(\mathrm{d} \psi-\cos \theta \mathrm{d} \phi)
$$

which is globally-defined (the factor of $q(y)$ is essential here). The contact structure is then easy to exhibit in terms of $\eta$ for the manifolds $Y^{p, q}[6]$. This theorem is the reason why one a priori requires $\operatorname{hcf}(p, q)=1$ - however see below.

\section{The Calabi-Yau cones}

It will be important for us to exploit the symplectic structure of the associated CalabiYau cones. Rather generally, the Calabi-Yau structure on the metric cone is specified by a Kähler (hence also symplectic) form $J$ and a holomorphic $(3,0)$ form $\Omega$, which in terms of the four-dimensional Kähler-Einstein data read as follows:

$$
\begin{aligned}
& J=r^{2} J_{4}+r \mathrm{~d} r \wedge\left(\frac{1}{3} \mathrm{~d} \psi^{\prime}+\sigma\right) \\
& \Omega=e^{i \psi^{\prime}} r^{2} \Omega_{4} \wedge\left[\mathrm{d} r+i r\left(\frac{1}{3} \mathrm{~d} \psi^{\prime}+\sigma\right)\right] .
\end{aligned}
$$

In the specific case of $C\left(Y^{p, q}\right)$, we have

$$
\begin{aligned}
J & =r^{2} \frac{1-y}{6} \sin \theta \mathrm{d} \theta \wedge \mathrm{d} \phi \\
& +\frac{1}{3} r \mathrm{~d} r \wedge(\mathrm{d} \psi-\cos \theta \mathrm{d} \phi)-\mathrm{d}\left(y r^{2}\right) \wedge\left(\mathrm{d} \alpha+\frac{1}{6}(\mathrm{~d} \psi-\cos \theta \mathrm{d} \phi)\right)
\end{aligned}
$$

and

$$
\begin{aligned}
\Omega & =e^{i \psi} r^{2} \sqrt{\frac{1-y}{6 w(y) q(y)}}(\mathrm{d} \theta+i \sin \theta \mathrm{d} \phi) \\
& \wedge\left[\mathrm{d} y-i w(y) q(y)\left(\mathrm{d} \alpha+\frac{1}{6}(\mathrm{~d} \psi-\cos \theta \mathrm{d} \phi)\right)\right] \\
& \wedge\left[\mathrm{d} r-2 i r\left(y \mathrm{~d} \alpha+(y-1) \frac{1}{6}(\mathrm{~d} \psi-\cos \theta \mathrm{d} \phi)\right)\right]
\end{aligned}
$$

where we used (2.18) (2.19) and have then rewritten the expressions in terms of the original coordinates.

Note that this calculation shows that $\Omega$ is invariant under $\partial / \partial \alpha$, namely

$$
\mathcal{L}_{\partial / \partial \alpha} \Omega=i_{\partial / \partial \alpha} \mathrm{d} \Omega+\mathrm{d}\left(i_{\partial / \partial \alpha} \Omega\right)=0
$$


implying that the Killing spinors are also invariant. This explicitly checks that upon performing a $T$-duality along the $\alpha$ direction to Type IIA string theory, the number of preserved supersymmetries is unchanged. In fact, this is obvious given the original construction [7] of these metrics. Since we are guaranteed existence of Killing spinors by the theorem of [34, and since we have now shown that the spinors are independent of $\alpha$, it follows that one may in fact take $\operatorname{hcf}(p, q)=h>1$ by taking a smooth quotient by $\mathbb{Z}_{h}$ of the simply-connected Sasaki-Einstein manifold $Y^{p / h, q / h}$. Since this is rather trivial, we take this as understood in the remainder of the paper.

\section{Complex coordinates}

It is easy to introduce a (local) set of complex coordinates. To do so we seek three closed complex one-forms $\eta^{i}$ such that $\Omega \wedge \eta^{i}=0$. First, consider the following local one-forms obeying the latter property:

$$
\begin{aligned}
\eta^{1} & =\frac{1}{\sin \theta} \mathrm{d} \theta+i \mathrm{~d} \phi \\
\tilde{\eta}^{2} & =\frac{1}{w(y) q(y)} \mathrm{d} y-i\left(\mathrm{~d} \alpha+\frac{1}{6}(\mathrm{~d} \psi-\cos \theta \mathrm{d} \phi)\right) \\
\tilde{\eta}^{3} & =\frac{\mathrm{d} r}{2 r}-i\left(\mathrm{~d} \alpha+(y-1)\left(\mathrm{d} \alpha+\frac{1}{6}(\mathrm{~d} \psi-\cos \theta \mathrm{d} \phi)\right)\right)
\end{aligned}
$$

where now

$$
\Omega=2 e^{i \psi} r^{3} \sqrt{\frac{\mathcal{Q}(y)}{3}} \sin \theta \eta^{1} \wedge \tilde{\eta}^{2} \wedge \tilde{\eta}^{3} .
$$

Taking $z_{1}=\tan \frac{\theta}{2} e^{i \phi}$ we immediately find

$$
\eta^{1}=\frac{\mathrm{d} z_{1}}{z_{1}}
$$

To obtain two more integrable one-forms one is free to consider linear combinations of the one-forms (2.27). Take

$$
\begin{aligned}
\eta^{2} & =-\frac{1}{6} \cos \theta \eta^{1}+\tilde{\eta}^{2} \\
\eta^{3} & =\frac{1}{6} \cos \theta \eta^{1}-y \tilde{\eta}^{2}+\tilde{\eta}^{3} .
\end{aligned}
$$

Notice that one can now simply drop the tildes in (2.28). Moreover the $\eta^{2}, \eta^{3}$ are now closed and hence locally exact. In particular

$$
\eta^{i}=\frac{\mathrm{d} z_{i}}{6 z_{i}}
$$


$i=2,3$, with

$$
\begin{aligned}
& z_{2}=\frac{1}{\sin \theta} \sqrt{\left(y-y_{1}\right)^{-\frac{1}{y_{1}}}\left(y_{2}-y\right)^{-\frac{1}{y_{2}}}\left(y_{3}-y\right)^{-\frac{1}{y_{3}}}} e^{-6 i \alpha-i \psi} \\
& z_{3}=r^{3} \sin \theta \sqrt{\mathcal{Q}(y)} e^{i \psi} .
\end{aligned}
$$

In terms of the $\left\{z_{i}\right\}$, the three-form assumes a very simple form:

$$
\Omega=\frac{1}{18 \sqrt{3}} \frac{\mathrm{d} z_{1} \wedge \mathrm{d} z_{2} \wedge \mathrm{d} z_{3}}{z_{1} z_{2}}
$$

\section{Supersymmetric cycles}

In this subsection we will show that the cones over the submanifolds $y=y_{1}, y_{2}$, which recall are the special orbits of the cohomogeneity one action, are in fact divisors in the Calabi-Yau cone. This amounts to showing that they are calibrated with respect to the four-form $\frac{1}{2} J \wedge J$. We denote the three-submanifolds as $\Sigma_{i}, i=1,2$, respectively.

Thus, we compute the pull-back of $\frac{1}{2} J \wedge J$ to the four-cycles in the Calabi-Yau cone $C\left(Y^{p, q}\right)$ specified by $y=y_{i}$. The latter are in fact cones over the Lens spaces $\Sigma_{1} \cong S^{3} / \mathbb{Z}_{p+q}, \Sigma_{2} \cong S^{3} / \mathbb{Z}_{p-q}$. We shall show in detail that this is indeed the topology in Section 4. However, this fact can also be seen by computing the pull-back of the Kähler form to the four-submanifolds. Defining $k=p+q, l=p-q$, these are ${ }^{10}$

$$
\begin{aligned}
\left.J\right|_{y=y_{1}} & =\ell y_{1}\left[-\frac{k}{2} r^{2} \sin \theta \mathrm{d} \theta \wedge \mathrm{d} \phi-r \mathrm{~d} r \wedge(\mathrm{d} 2 \gamma-k \cos \theta \mathrm{d} \phi)\right] \\
\left.J\right|_{y=y_{2}} & =\ell y_{2}\left[\frac{l}{2} r^{2} \sin \theta \mathrm{d} \theta \wedge \mathrm{d} \phi-r \mathrm{~d} r \wedge(\mathrm{d} 2 \gamma+l \cos \theta \mathrm{d} \phi)\right]
\end{aligned}
$$

and are precisely the Kähler forms associated to cones over round Lens spaces $S^{3} / \mathbb{Z}_{k}$ and $S^{3} / \mathbb{Z}_{l}$, respectively. Indeed, since $\gamma$ has period $2 \pi$, the one-forms multiplying $\mathrm{d} r$ are precisely global angular forms (global connections) on the total spaces of circle bundles over $S^{2}$ with Chern numbers $k$ and $-l$, respectively. The total spaces of such bundles are precisely $S^{3} / \mathbb{Z}_{k}$ and $S^{3} / \mathbb{Z}_{l}$, respectively. From these expressions, one calculates

$$
\left.\frac{1}{2} J \wedge J\right|_{y=y_{i}}=\frac{r^{3} \ell y_{i}\left(1-y_{i}\right)}{3} \sin \theta \mathrm{d} \theta \wedge \mathrm{d} \phi \wedge \mathrm{d} \gamma \wedge \mathrm{d} r
$$

Let us compare this with the volume form induced on $\Sigma_{i}$ from the metric (2.4). This is given by

$$
\text { vol }=\frac{r^{3} \ell \sqrt{w\left(y_{i}\right)}\left(1-y_{i}\right)}{6} \sin \theta \mathrm{d} \theta \wedge \mathrm{d} \phi \wedge \mathrm{d} \gamma \wedge \mathrm{d} r
$$

\footnotetext{
${ }^{10}$ Recall that $y_{1}<0$ and $y_{2}>0$.
} 
Remarkably, since $w\left(y_{i}\right)=4 y_{i}^{2}$ at any root of the cubic (2.7) we see that this precisely agrees with (2.36). Thus we see that both $C\left(\Sigma_{1}\right)=\left\{y=y_{1}\right\}$ and $C\left(\Sigma_{2}\right)=\left\{y=y_{2}\right\}$ are divisors of $C\left(Y^{p, q}\right)$, or in other words they are supersymmetric submanifolds.

We may now write down the volumes of the $\Sigma_{i}$. Here one needs to use the explicit formulae for the roots of the cubic $y_{1}$ and $y_{2}$ in terms of $p$ and $q$ :

$$
\begin{aligned}
& y_{1}=\frac{1}{4 p}\left(2 p-3 q-\sqrt{4 p^{2}-3 q^{2}}\right) \\
& y_{2}=\frac{1}{4 p}\left(2 p+3 q-\sqrt{4 p^{2}-3 q^{2}}\right) .
\end{aligned}
$$

One then easily calculates

$$
\begin{aligned}
& \operatorname{vol}\left(\Sigma_{1}\right)=\frac{q^{2}(p+q)\left(-2 p+3 q+\sqrt{4 p^{2}-3 q^{2}}\right)^{2}}{2 p^{2}\left(3 q^{2}-2 p^{2}+p \sqrt{4 p^{2}-3 q^{2}}\right)^{2}} \pi^{2} \\
& \operatorname{vol}\left(\Sigma_{2}\right)=\frac{q^{2}(p-q)\left(2 p+3 q-\sqrt{4 p^{2}-3 q^{2}}\right)^{2}}{2 p^{2}\left(3 q^{2}-2 p^{2}+p \sqrt{4 p^{2}-3 q^{2}}\right)^{2}} \pi^{2} .
\end{aligned}
$$

In particular, let us write down the volumes of $\Sigma_{i}$ in the case of $p=2, q=1$ :

$$
\operatorname{vol}\left(\Sigma_{1}\right)=\frac{\pi^{2}}{108}(31+7 \sqrt{13}), \quad \operatorname{vol}\left(\Sigma_{2}\right)=\frac{\pi^{2}}{36}(7+\sqrt{13}) .
$$

\section{Moment maps and convex rational polyhedral cones}

In the remainder of this paper it will be crucial for us that the Sasaki-Einstein manifolds $Y^{p, q}$ admit an effectively acting three-torus $\mathbb{T}^{3}=U(1)^{3}$ of isometries, which moreover is Hamiltonian. The latter means that the action preserves the symplectic form of the cone $C\left(Y^{p, q}\right)$ and that one can use this to introduce a moment map. The torus is just the maximal torus in the isometry group, and the fact that the torus is half the dimension of the cone means that, by definition, the cones are toric. The image of the cone under the corresponding moment map generally belongs to a special class of convex rational polyhedral cones in $\mathbb{R}^{3}$ [35, 20, - these are simply convex cones formed by intersecting some number of planes through the origin. The normal vectors to these planes, or facets, are necessarily rational and describe which $U(1)$ subgroup of $\mathbb{T}^{3}$ is vanishing over the corresponding codimension two submanifold of $C\left(Y^{p, q}\right)$. 
This generalises the well-known result in symplectic geometry that the image of the moment map for a compact toric symplectic manifold is always a particular type of convex rational polytope called a Delzant polytope.

In this section we give a general review of symplectic toric geometry. This is mainly rather standard material from the point of view of a symplectic geometer - the reader who is familiar with this subject may therefore wish to skip this section. On the other hand, we hope that this will be a useful self-contained presentation of the material.

\subsection{Moment maps for torus actions}

In this subsection we give a general summary of moment maps, Hamiltonian torus actions, and symplectic toric manifolds, orbifolds and cones, together with the properties of their images under the moment maps, which are always particular types of rational polytopes (or polyhedral cones) in $\mathbb{R}^{n}$. The case of compact manifolds [36. 37, 21] is rather standard in symplectic geometry, but the generalisation for orbifolds [38, and especially cones [35, 20], is quite recent.

We begin by giving a general definition. Suppose that the torus $\mathbb{T}^{n}$ acts effectively - meaning that every non-trivial element moves at least one point - on a symplectic manifold $M$ with symplectic form $\omega$. We identify the Lie algebra of this torus, as well as its dual, with Euclidean $n$-space, so $\mathrm{t}_{n} \equiv \operatorname{Lie}\left(\mathbb{T}^{n}\right) \cong \mathbb{R}^{n}, \mathrm{t}_{n}^{*} \cong \mathbb{R}^{n}$. Then a moment map for the torus action is simply a $\mathbb{T}^{n}$-invariant map

$$
\mu: M \rightarrow \mathrm{t}_{n}^{*} \cong \mathbb{R}^{n}
$$

satisfying the condition

$$
\left.\mathrm{d} \mu^{i}=V^{i}\right\lrcorner \omega
$$

Here $V^{i}$ denotes the vector field on $M$ corresponding to the basis vector $e_{i}$ in $\mathrm{t}_{n} \cong \mathbb{R}^{n}$, and $\mu^{i}$ denotes the component of the map $\mu$ in the direction $e_{i}$ i.e. $\mu=\left(\mu^{1}, \ldots, \mu^{n}\right)$. Clearly this moment map is unique only up to an additive integration constant.

To see where this map comes from, suppose for simplicity that one has a $U(1)$ action on a symplectic manifold $M$, generated by some vector field $V$, which moreover preserves the symplectic form. One then says that the $U(1)$ action is symplectic. The latter means that

$$
\mathcal{L}_{V} \omega=0
$$

where $\mathcal{L}$ is the Lie derivative. Since $\omega$ is closed, this condition is just

$$
\mathrm{d}(V\lrcorner \omega)=0 .
$$


As long as the closed one-form $V\lrcorner \omega$ is trivial as a cohomology class, $[V\lrcorner \omega]=0 \in$ $H^{1}(M ; \mathbb{R})$, then one can "integrate" this equation to a function $\mu$, which is precisely the moment map for the $U(1)$ action. The action is then said to be Hamiltonian. For example, the $U(1)$ which rotates one of the circles in $\mathbb{T}^{2}$, with obvious symplectic form, is not Hamiltonian. Clearly, if $H^{1}(M ; \mathbb{R})$ is trivial then all symplectic actions are in fact Hamiltonian. A symplectic toric manifold is then by definition a symplectic manifold of dimension $2 n$ with an effective Hamiltonian torus action by $\mathbb{T}^{n}$.

It is by now a classic fact in symplectic geometry that, for a compact symplectic toric manifold $M$, the image of $M$ under $\mu$ is a certain kind of convex rational polytope in $\mathbb{R}^{n}$ called a Delzant polytope [21. Recall that a polytope is just the convex hull of some finite number of points in $\mathbb{R}^{n}$. The codimension one hyperplanes that bound the polytope are called its facets. The symplectic toric manifold is then a torus fibration over this polytope, with the fibres collapsing in a certain way over the facets. More precisely, over an interior point of the polytope the fibre of the moment map (the inverse image of the point) is the whole torus $\mathbb{T}^{n}$, but over the boundary facets this fibre collapses to $\mathbb{T}^{n-1} \cong \mathbb{T}^{n} / U(1)$. Such a $U(1)$ subgroup is specified by a vector in the weight lattice $v \in \mathbb{Z}^{n}$ of $\mathbb{T}^{n}$, and this vector is in fact just the normal vector to the facet. Moreover the $U(1)$ fixes a corresponding codimension two submanifold of $M$. To see this, consider the case where $v=e_{1}=(1,0, \ldots, 0)$. Denote the corresponding vector field as $V$. Then over a codimension two fixed point set $F \subset M$ we have that $V=0$, and moreover $F$ is itself symplectic toric with respect to the torus $\mathbb{T}^{n-1} \cong \mathbb{T}^{n} / U(1)$. In particular, the moment map $\mu$ restricted to $F$ is constant in the direction corresponding to $V$ i.e. $\mu^{1}=c=$ constant. Then $\left.\mu_{F} \equiv \mu\right|_{F}$ is a moment map for $F$ with $\left\langle\mu_{F}, e_{1}\right\rangle=c$. This defines the hyperplane at $x_{1}=c$, where $\left\{x_{i}\right\}, i=1, \ldots, n$ are coordinates on $\mathbb{R}^{n}$. The general case follows similarly. The normal vectors to the facets are thus all rational vectors. If two facets intersect over a codimension two face in $\mathbb{R}^{n}$, then both the corresponding $U(1)$ 's vanish, and the fibre over this face is a $\mathbb{T}^{n-2}$. Continuing in this way, the vertices of the polytope are precisely the points in $M$ which are fixed under the entire torus action. The fact that the polytope is always convex follows from an argument using Morse theory [36. 37.

Delzant polytopes satisfy some additional conditions, as well as being rational:

- simplicity - $n$ edges meet at each vertex.

- smoothness - for each vertex, the corresponding $n$ edge vectors $u_{i}, i=1, \ldots, n$ 
form a $\mathbb{Z}$-basis ${ }^{11}$ of $\mathbb{Z}^{n}$.

The polytope data is sufficient to recover the original symplectic toric manifold. Moreover, the correspondence between Delzant polytopes and compact symplectic toric manifolds is one-to-one. Thus, to any Delzant polytope $\Delta$ one can associate a corresponding symplectic toric manifold whose image under the moment map is precisely $\Delta$. The proof of this is by construction. This will be extremely important for us in Section 5 - in physics terms, the construction realises the manifold as the vacuum of a gauged linear sigma model [22].

We now briefly explain the above conditions. Assuming the first condition holds, the second condition avoids orbifold singularities. Indeed if the smoothness condition fails then $\mathbb{T}^{n} /<u_{i}>\cong \Gamma$ is a non-trivial finite abelian group, where $<u_{i}>$ denotes the span of the $u_{i}$ over $\mathbb{Z}$. In this case the corresponding point in $M$ is an orbifold point with structure group $\Gamma$. Indeed, there is a corresponding classification of symplectic toric orbifolds where the smoothness condition is dropped, and moreover one attaches to each facet a positive integer label 38. This latter necessity can be seen by considering the weighted projective space $\mathbb{C P}_{[k, l]}^{1}$. This is topologically a sphere, with neighbourhoods of the north and south poles replaced by orbifold singularities $\mathbb{C} / \mathbb{Z}_{k}$ and $\mathbb{C} / \mathbb{Z}_{l}$, respectively. The quotient by the $U(1)$ action which rotates around the equator is clearly just a line segment. Thus the orbifold information is completely lost when one takes the image under the moment map. To remedy this 38, quite generally, one associates to each facet a positive integer label $m$, such that the preimage of any point in that facet has local orbifold structure group $\mathbb{Z}_{m}$. In the case at hand, the endpoints of the interval are assigned labels $k$ and $l$, respectively.

The first condition - simplicity - avoids even worse singularities than orbifold singularities. As we shall see, for symplectic toric cones this condition is not satisfied at the vertex corresponding to the apex of the cone, unless of course the cone is in fact an orbifold singularity.

\subsection{Toric Calabi-Yau cones}

This brings us to the generalisation of this theorem [35, 20] for symplectic toric cones, which is the case of interest for us. These may be regarded as non-compact symplectic toric manifolds with a homothetic action of $\mathbb{R}^{+}$which commutes with the torus action and acts by rescaling the symplectic form. In fact, every symplectic toric cone is a

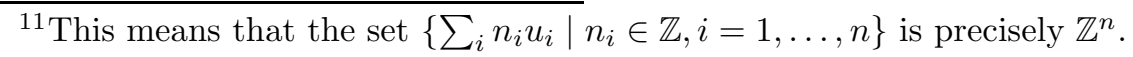


cone over a toric contact manifold $Y$, and vice versa. In this case the moment map for the symplectic toric cone $C(Y)=\mathbb{R}^{+} \times Y$ may still be defined, away from the apex of the cone, and takes a special form. Define the one-form

$$
\left.\eta_{C}=r \partial / \partial r\right\lrcorner \omega
$$

where $r \partial / \partial r$ is the Euler vector, which generates the $\mathbb{R}^{+}$action on the cone, and $\omega$ is the symplectic form. Identifying the base of the cone $Y=\left.C(Y)\right|_{r=1}$ we may define the one-form ${ }^{12} \eta=\left.\eta_{C}\right|_{r=1}$. One then easily sees that

$$
\omega=r \mathrm{~d} r \wedge \eta+\frac{1}{2} r^{2} \mathrm{~d} \eta
$$

A straightforward calculation then shows that the moment map $\mu$ on the cone is given by

$$
<\mu, e_{i}>=\eta_{C}\left(V^{i}\right)
$$

for any basis vector $e_{i}$ of $\mathrm{t}_{n}$ and corresponding vector field $V^{i}$. Here $\eta_{C}\left(V^{i}\right)$ just denotes the dual pairing between one-forms and vectors. The choice of integration constant makes this moment map transform homogeneously under the $\mathbb{R}^{+}$homothetic action. It also ensures that the apex of the cone, at $r=0$, is mapped to the origin of $\mathbb{R}^{n}$.

Let us now also assume ${ }^{13}$ that the symplectic toric cones are of Reeb type. This means that there is some element $\zeta \in \mathrm{t}_{n} \cong \mathbb{R}^{n}$ such that $\langle\mu, \zeta\rangle$ is a strictly positive function on $C(Y)$. The image of the moment map is then a strictly convex rational polyhedral cone in $\mathbb{R}^{n}$ [35], which, moreover, is good in the sense of reference [20]. Recall that a rational polyhedral cone may be defined as a set of points in $\mathbb{R}^{n}$ of the form

$$
C=\left\{x \in \mathbb{R}^{n} \mid<x, v_{i}>\leq 0, i=1, \ldots, d\right\}
$$

where the rational vectors $v_{i}$ are the outward pointing normal vectors to the facets of the cone $C$. Here we may assume that the set $\left\{v_{i}\right\}$ is minimal, meaning that one cannot drop any vector $v_{i}$ from the definition without changing the cone, and also primitive - recall that a vector with integral entries is said to be primitive if it cannot be written as $n v$ where $1 \neq n \in \mathbb{Z}$ and $v$ is also a vector with integral entries. The

\footnotetext{
${ }^{12}$ More precisely we embed $Y$ in $C(Y)$ at $r=1$ and then pull back $\eta_{C}$ to $Y$ to give $\eta$.

${ }^{13}$ The symplectic toric cones that are not of Reeb type are rather uninteresting: they are either cones over $S^{2} \times S^{1}$, cones over principle $\mathbb{T}^{3}$ bundles over $S^{2}$, or cones over products $\mathbb{T}^{m} \times S^{m+2 j-1}$, $m>1, j \geq 0$ [20.
} 
requirement that this polyhedral cone is strictly convex means that it is a cone over a polytope.

The "conelike" nature of the subspace (3.8) of course descends from the "conelike" nature of the cone we began with - the property that $C(Y)$ is invariant under a group $\mathbb{R}^{+}$of homotheties will be inherited by the image under the moment map since by definition the moment map commutes with the $\mathbb{R}^{+}$action. Clearly the simplicial condition will fail at the apex $=$ origin of $\mathbb{R}^{n}$ unless $d=n$. Moreover, even in this case the smoothness condition will fail unless the edges span $\mathbb{Z}^{n}$. In this case, by an $S L(n ; \mathbb{Z})$ transformation of the torus, one can take this to be the standard basis, whence it is easy to see that the cone one started with is just $\mathbb{R}^{2 n}$ with its usual symplectic structure. This latter point brings up an issue worth stressing: one is of course free to make an $S L(n ; \mathbb{Z})$ transformation of the torus $\mathbb{T}^{n}$ resulting in a change of the basis $e_{i}$. This will generate a corresponding $S L(n ; \mathbb{Z})$ transformation on the image under the moment map. Thus the polytopes and polyhedral cones are only unique up to such transformations.

As shown in [20], the image of a symplectic toric cone under its moment map is also a good polyhedral cone. This means the following. Let $\mathcal{F}$ be a proper face of the cone $C$. Over this face there will be a corresponding torus $\mathbb{T}_{\mathcal{F}} \subset \mathbb{T}^{n}$ which is collapsing to zero. For example, in the case that $\mathcal{F}$ is a facet, $\mathbb{T}_{\mathcal{F}} \cong U(1)$. For a face $\mathcal{F}$ of codimension $m$ the torus is dimension $m: \operatorname{dim} \mathbb{T}_{\mathcal{F}}=m$. Now, the torus $\mathbb{T}_{\mathcal{F}} \subset \mathbb{T}^{n}$ determines a lattice $\mathbb{Z}_{\mathbb{T}_{\mathcal{F}}}=\operatorname{ker}\left(\exp : \mathrm{t}_{\mathcal{F}} \rightarrow \mathbb{T}_{\mathcal{F}}\right) \subset \mathbb{Z}^{n}$. We then require that the corresponding collection of normal vectors form an integral basis for this lattice, i.e. the collection of normal vectors span the lattice $\mathbb{Z}_{\mathbb{T}_{\mathcal{F}}}$ over $\mathbb{Z}$. This condition may be regarded as a generalisation of Delzant's conditions for symplectic toric manifolds to symplectic toric cones.

In the particular case where the symplectic cone came from a Calabi-Yau cone, one has additional information. In particular, the Sasaki-Einstein metric on $Y$ may be used to define the dual vector field $K$ with $\eta(K)=1$. This is called the Reeb vector in the language of contact geometry. Physically this is dual to the R-symmetry of the field theory. Then there is a corresponding Lie algebra element $\zeta \in \mathrm{t}_{n}$, and we have

$$
<\mu_{Y}, \zeta>=\eta(K)=1
$$

It follows that the image $\mu_{Y}(Y)$ lies in the above hyperplane, which is called the characteristic hyperplane [39]. In particular, note that the polytope one obtains by 
intersecting the polyhedral cone with the characteristic hyperplane will be rational if and only if $\zeta$ is rational. The latter condition is required precisely for quasi-regularity of the Sasaki-Einstein metric. Correspondingly, this is also the condition that the characteristic polytope satisfies for an orbifold polytope, and thus that the quotient of $Y$ by the $U(1)$ action generated by $K$ gives an orbifold. Notice that one may then apply the modified Delzant construction of [38] to obtain a gauged linear sigma model describing this orbifold. In principle one could do this for our quasi-regular Sasaki-Einstein manifolds, although we will not pursue this here.

\section{The moment map and its image}

In this section we explicitly construct the polyhedral cone corresponding to the image of $C\left(Y^{p, q}\right)$ under its moment map.

The Calabi-Yau cones on $Y^{p, q}$ are symplectic toric cones. In particular, the $\mathbb{T}^{3}$ action, which is the maximal torus of the isometry group, is Hamiltonian, and one can explicitly integrate the symplectic form (2.24) to obtain a moment map. Note in fact that (2.24) can be written as

$$
J=\mathrm{d} \phi \wedge \mathrm{d}\left[r^{2} \frac{1-y}{6} \cos \theta\right]+\mathrm{d} \psi \wedge \mathrm{d}\left[-r^{2} \frac{1-y}{6}\right]+\mathrm{d} \gamma \wedge \mathrm{d}\left[\ell r^{2} y\right] .
$$

The torus $\mathbb{T}^{3}$ is essentially generated by the Killing vectors $\partial / \partial \phi, \partial / \partial \psi, \partial / \partial \gamma$. However, one must be careful to ensure that the Killing vectors one takes really do form a basis for an effectively acting $\mathbb{T}^{3}$. Since this is a slightly subtle point, we first explain a simpler example.

\section{A brief detour on Lens spaces}

Let us consider the Lens spaces $L(1, m)=S^{3} / \mathbb{Z}_{m}$ where we regard $S^{3}$ as a (squashed) Hopf $S^{1}$ fibration over a round two-sphere. The isometry groups of the latter may be analysed as follows. Embed the round sphere $S^{3}$ in $\mathbb{R}^{4}$, and regard $\mathbb{R}^{4} \cong \mathbb{H}$ as the space of quaternions. The isometry group of $S^{3}$, preserving its orientation, is $S O(4) \cong\left(S U(2)_{L} \times S U(2)_{R}\right) / \mathbb{Z}_{2}$, where $S U(2)_{L, R}$ denote left and right actions by the unit quaternions $S p(1) \cong S U(2)$. Thus $\mathbb{H} \ni q \rightarrow a q b^{-1}$ where $(a, b) \in$ $S U(2) \times S U(2) \cong \operatorname{Spin}(4)$. Notice that $(-1,-1)$ acts trivially, i.e. the two $S U(2)$ factors intersect precisely over the antipodal map. Thus, for a squashed three-sphere, meaning that one squashes the Hopf $S^{1}$ fibre relative to the base round $S^{2}$, we see that the isometry group is $U(2) \cong(S U(2) \times U(1)) / \mathbb{Z}_{2}$. 
However, suppose we now take a quotient of $\mathbb{R}^{4} \cong \mathbb{H}$ on the right by $\mathbb{Z}_{m} \subset U(1)$. One still has a left $S U(2)$ action and a right $U(1)$ action, where the latter now factors through a cyclic group of order $m$. For example, take $m=2$, thus giving $S^{3} / \mathbb{Z}_{2} \cong \mathbb{R P}^{3}$. In complex coordinates, $\mathbb{H} \cong \mathbb{C} \oplus \mathbb{C}$, this means $\left(z_{1}, z_{2}\right) \sim\left(-z_{1},-z_{2}\right)$ which identifies antipodal points on the three-sphere. It follows that the centre of $S U(2)_{L}$ acts trivially and hence the effectively acting isometry group is $S O(3) \times U(1)$, where $U(1)$ rotates the $S^{1}$ fibre with weight one - half the weight of $U(1) \subset S U(2)_{R}$. It now follows that the isometry group for $S^{3} / \mathbb{Z}_{m}$ for all odd $m$ is $U(2)$, whereas for even $m$ it is $S O(3) \times U(1)$ - it is precisely the even cases where $\mathbb{Z}_{m}$ contains the antipodal map above.

Clearly these Lens spaces have an isometric $\mathbb{T}^{2}$ action. Take $m=2 r$. From our discussion above, if $V_{1}$ denotes the Killing vector that rotates the $S^{2}$ about its equator with weight one, and $V_{2}$ denotes the Killing vector that rotates the $S^{1}$ fibre, also with weight one, then $V_{1}, V_{2}$ do indeed form a basis for an effectively acting $\mathbb{T}^{2}$. This is the obvious $\mathbb{T}^{2}$ in $S O(3) \times U(1)$.

For $m=2 r+1$ one needs to be more careful: the isometry group is $U(2)$. For example, for $r=0$ one has the unit chiral spin bundle of $S^{2}$. As is well-known, a single rotation of $S^{2}$ will not result in the spinor coming back to itself: one needs to rotate twice. For an effective action one should thus take a basis $e_{1}=V_{1}+\frac{1}{2} V_{2}$, $e_{2}=V_{2}$. Here $e_{1}$ is half the generator of the diagonal $U(1)$ in $S U(2) \times U(1)$, and $V_{2}$ generates the $U(1)$ factor.

Of course, one can use the basis $e_{1}=V_{1}+\frac{m}{2} V_{2}, e_{2}=V_{2}$ quite generally in all cases. Indeed, recall that the choice of basis is unique only up to an $S L(2 ; \mathbb{Z})$ transformation. For $m=2 r$ even, this basis is just the $S L(2 ; \mathbb{Z})$ transformation

$$
\left(\begin{array}{ll}
1 & r \\
0 & 1
\end{array}\right)
$$

of the basis $\left\{V_{1}, V_{2}\right\}$.

\section{The moment cone}

After this brief digression, we return to the case of interest. First let us note from the results above that the isometry group of the base $B$ is $S O(3) \times U(1)$. Indeed, for fixed $y, y_{1}<y<y_{2}$, we have a copy of $S^{3} / \mathbb{Z}_{2} \cong \mathbb{R P}^{3}$, and the group $S O(3) \times U(1)$ acts with cohomogeneity one on $B$ with fixed $y$ as generic orbit. Thus, in particular, we may take a basis $\partial / \partial \phi+\partial / \partial \psi, \partial / \partial \phi$ for an effectively acting two-torus. For 
$C\left(Y^{p, q}\right)$, one must also add the direction $\partial / \partial \gamma$. However, here one must be careful to ensure the orbits of the vectors close, and that this torus then acts effectively, just as for the Lens spaces. One finds the following choice suffices:

$$
\begin{aligned}
e_{1} & =\frac{\partial}{\partial \phi}+\frac{\partial}{\partial \psi} \\
e_{2} & =\frac{\partial}{\partial \phi}-\frac{l}{2} \frac{\partial}{\partial \gamma} \\
e_{3} & =\frac{\partial}{\partial \gamma} .
\end{aligned}
$$

Recall that the submanifolds $y=y_{1}, y=y_{2}$ of $Y^{p, q}$ are Lens spaces $S^{3} / \mathbb{Z}_{k}, S^{3} / \mathbb{Z}_{l}$, respectively, where recall $k=p+q, l=p-q$ - the shift in $e_{2}$ is then required precisely by the reasoning above. Note that one can replace $l$ in the formula for $e_{2}$ by anything congruent to $l$ modulo two (for example, $k$ ) - this is just an $S L(3 ; \mathbb{Z}$ ) transformation of the torus. Also note that for $l$ even one can in fact take a basis $\partial / \partial \phi, \partial / \partial \psi, \partial / \partial \gamma$. The effectively acting isometry group is thus $S O(3) \times U(1) \times U(1)$ in this case. For $l$ odd this becomes $U(2) \times U(1)$.

Let us now consider the moment map for $C\left(Y^{p, q}\right)$. In terms of the basis $e_{1}, e_{2}, e_{3}$ above one finds:

$$
\vec{\mu}=\left(\frac{r^{2}}{6}(1-y)(\cos \theta-1), \frac{r^{2}}{6}(1-y) \cos \theta-\frac{r^{2}}{2} l \ell y, \ell r^{2} y\right) .
$$

Notice that this involves the generically irrational parameter $\ell$.

We will now describe the image of $\vec{\mu}$, and check that it is given by a good convex rational polyhedral cone in $\mathbb{R}^{3}$, as predicted by the results of [35, 20. First, note that the edges of the cone can be identified by fixing any non-zero value of $r$, say $r=1$, and then finding the submanifolds which are fixed under some $\mathbb{T}^{2} \subset \mathbb{T}^{3}$ action. Indeed, the edges of the cone, which generate it, are precisely the images of submanifolds in $C\left(Y^{p, q}\right)$ over which some two-torus collapses. There are four such submanifolds at $r=1$, given by the north $(N)$ and south $(S)$ poles of the base and fibre twospheres: these are all copies of a circle - specifically, the fibre over the corresponding point on the base $B$. We denote the subspaces as follows: $N N=\left\{y=y_{2}, \theta=0\right\}$, $N S=\left\{y=y_{2}, \theta=\pi\right\}, S N=\left\{y=y_{1}, \theta=0\right\}, S S=\left\{y=y_{1}, \theta=\pi\right\}$. Then, using 
the useful relations ${ }^{14}$

$$
\begin{aligned}
& 1-y_{1}=-3 \ell k y_{1} \\
& 1-y_{2}=3 \ell l y_{2}
\end{aligned}
$$

we find (at $r=1$ )

$$
\begin{aligned}
\vec{\mu}(N N) & =\ell y_{2}(0,0,1) \\
\vec{\mu}(N S) & =\ell y_{2}(-l,-l, 1) \\
\vec{\mu}(S N) & =\ell y_{1}(0,-p, 1) \\
\vec{\mu}(S S) & =\ell y_{1}(k, q, 1) .
\end{aligned}
$$

Note that the irrational parameter $\ell$ has factored out and the vectors in (4.6) represent four lines which are spanned as $r$ varies from 0 to infinity. Noting that $y_{1}<0$ and $y_{2}>0$ it is then easy to verify that these are the edges of a four-faceted polyhedral cone in $\mathbb{R}^{3}$ generated by:

$$
u_{1}=[0, p,-1], \quad u_{2}=[-k,-q,-1], \quad u_{3}=[0,0,1], \quad u_{4}=[-l,-l, 1]
$$

with outward-pointing primitive normals:

$$
v_{1}=[1,0,0], \quad v_{2}=[1,-2,-l], \quad v_{3}=[1,-1,-p], \quad v_{4}=[1,-1,0] .
$$

As described above, these normals characterise codimension two fixed point sets in $C\left(Y^{p, q}\right)$ over which a circle of the three-torus shrinks to zero size. The corresponding linear combination of Killing vectors in $[\partial / \partial \phi, \partial / \partial \psi, \partial / \partial \gamma]$ should then have vanishing norm when restricted to the pre-image of the facet. Indeed, using the metric (2.4) it is straightforward to verify that the four Killing vectors

$$
V_{1}=\frac{\partial}{\partial \phi}+\frac{\partial}{\partial \psi}, \quad V_{2}=-\frac{\partial}{\partial \phi}+\frac{\partial}{\partial \psi}, \quad V_{3}=\frac{\partial}{\partial \psi}-\frac{k}{2} \frac{\partial}{\partial \gamma}, \quad V_{4}=\frac{\partial}{\partial \psi}+\frac{l}{2} \frac{\partial}{\partial \gamma}
$$

vanish on the submanifolds given by $\theta=0, \theta=\pi, y=y_{1}$, and $y=y_{2}$ respectively. Note that the normals obtained with the moment map use only the symplectic structure of the manifolds, whereas the norms of the Killing vectors are computed using the metrics.

Let us now make the following observations about the normal vectors $v_{1}, \ldots, v_{4}$ :

\footnotetext{
${ }^{14}$ One can derive these using the the explicit form for the periods $P_{i}$ of Section 2 , after using the cubic equation (2.7).
} 
- $\left\{v_{1}, \ldots, v_{4}\right\}$ span $\mathbb{Z}^{3}$ over $\mathbb{Z}$. Indeed it is trivial to see that $\left\langle v_{1}, v_{4}\right\rangle=<$ $\left.E_{1}, E_{2}\right\rangle$. The direction $\left\langle E_{3}\right\rangle$ is then obtained as a linear combination of $v_{1}, v_{2}, v_{3}, v_{4}$. Indeed, since hcf $(l, p)=1$ by Euclid's algorithm there are integers $a, b \in \mathbb{Z}$ such that $a l+b p=1$.

- For each of the four edge vectors $u_{i}, i=1, \ldots, 4$, the corresponding two normal vectors $v_{i_{1}}, v_{i_{2}}, i_{1} \neq i_{2} \in\{1234\}$ with $u_{i} \cdot v_{i_{1}}=u_{i} \cdot v_{i_{2}}=0$ satisfy

$$
\left\{a_{1} v_{i_{1}}+a_{2} v_{i_{2}} \mid a_{1}, a_{2} \in \mathbb{R}\right\} \cap \mathbb{Z}^{3}=\left\{a_{1} v_{i_{1}}+a_{2} v_{i_{2}} \mid a_{1}, a_{2} \in \mathbb{Z}\right\} .
$$

The second condition is precisely the condition that the cone is good, in the sense of reference [20]. Indeed, this must be true since in [20] it is shown that the image of a symplectic toric cone under its moment map is always a good rational polyhedral cone. The first property does not generically hold, but is special to the geometries we are considering. As we will see later, it is related to the fact that the Sasaki-Einstein manifolds we began with are simply-connected.

It will be useful to know the topology of the codimension two submanifolds. Let us denote them as $F_{i}$, where $i=1, \ldots, 4$, respectively. Explicitly we have $F_{1}=\{\theta=0\}$, $F_{2}=\{\theta=\pi\}, F_{3}=\left\{y=y_{1}\right\}, F_{4}=\left\{y=y_{2}\right\}$. If we project out the $\gamma$ direction, these are all copies of $S^{2}$. The first two, $F_{1} / U(1), F_{2} / U(1)$, are the two fibres of $B=S^{2} \hookrightarrow S^{2}$ over the north and south poles of the base $S^{2}$, and so are representatives of the cycle $C_{1}$. The third and fourth, $F_{3} / U(1), F_{4} / U(1)$, are the sections of the $S^{2}$ bundle at the south and north poles of the fibre $S^{2}$, respectively ${ }^{15}$. Since the $\gamma$ direction describes a principle $U(1)$ bundle over each of these spheres, the total spaces $F_{i}$ will be Lens spaces $L(1, m) \cong S^{3} / \mathbb{Z}_{m}$ for various values of $m \in \mathbb{Z}$. To see which Lens spaces one has, one can simply integrate the curvature two-form $\ell^{-1} \mathrm{~d} A$ over $F_{i} / U(1)$ for each $i=1, \ldots, 4$. One finds

$$
F_{1} \cong F_{2} \cong S^{3} / \mathbb{Z}_{p}, \quad F_{3} \cong S^{3} / \mathbb{Z}_{k}, \quad F_{4} \cong S^{3} / \mathbb{Z}_{l}
$$

Thus the facets of the polyhedral cone lift to cones over the above four Lens spaces. The latter two are calibrated submanifolds, as we saw in Section 2.

\section{Gauged linear sigma models}

In this section we begin by giving a brief review of gauged linear sigma models [22]. We then move on to describe Delzant's construction [21] which from a polytope

\footnotetext{
${ }^{15}$ These were denoted $S_{1}$ and $S_{2}$ in [6].
} 
$\Delta$ constructs a gauged linear sigma model whose vacuum manifold is precisely the symplectic toric manifold corresponding to $\Delta$. The construction also goes through for cones, provided one starts with a good convex rational polyhedral cone [20. We then use this method to construct the sigma model for the cone $C\left(Y^{p, q}\right)$. Using this approach, turning on Fayet-Iliopoulos parameters in the linear sigma model one (partially) resolves the conical singuarity. As a check on our result, we explicitly show how one can recover the topology and group action on $Y^{p, q}$ from the linear sigma model description, thus closing the loop of arguments. This is summarised below:

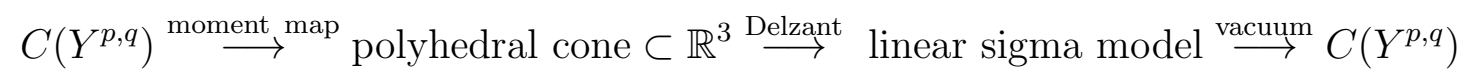

\subsection{A brief review}

Let $z_{1}, \ldots, z_{d}$ denote complex coordinates on $\mathbb{C}^{d}$. In physics terms these will be the lowest components of chiral superfields $\Phi_{i}, i=1, \ldots, d$. We may specify an action of the group $\mathbb{T}^{r} \cong U(1)^{r}$ on $\mathbb{C}^{d}$ by giving the integral charge matrix $Q=\left\{Q_{a}^{i} \mid i=\right.$ $1, \ldots, d ; a=1, \ldots, r\}$; here the $a$ th copy of $U(1)$ acts on $\mathbb{C}^{d}$ as

$$
\left(z_{1}, \ldots, z_{d}\right) \rightarrow\left(\lambda^{Q_{a}^{1}} z_{1}, \ldots, \lambda^{Q_{a}^{d}} z_{d}\right)
$$

where $\lambda \in U(1)$. We may then perform the Kähler quotient $X=\mathbb{C}^{d} / / U(1)^{r}$ by imposing the $r$ constraints

$$
\sum_{i=1}^{d} Q_{a}^{i}\left|z_{i}\right|^{2}=t_{a} \quad a=1, \ldots, r
$$

where $t_{a}$ are constants, and then quotienting out by $U(1)^{r}$. The resulting space has complex dimension $n=d-r$ and inherits a Kähler structure, and thus also a symplectic structure, from that of $\mathbb{C}^{d}$. In physics terms, the constraints (5.2) correspond to setting the $D$-terms of the gauged linear sigma model to zero to give the vacuum, where $t_{a}$ are Fayet-Iliopoulos parameters - one for each $U(1)$ factor. The quotient by $\mathbb{T}^{r}$ then removes the gauge degrees of freedom. Thus the Kähler quotient of the gauged linear sigma model precisely describes the classical vacuum of the theory. Note that the Kähler class of the quotient $X$ depends linearly on the FI parameters $t_{a}$, and moreover even the topology of the quotient will depend on these. Also observe that, setting all $t_{a}=0$, the resulting quotient will be a cone. One sees this by noting that $z_{i} \rightarrow \nu z_{i}, i=1, \ldots, d$ is a symmetry in this case, where $\nu \in \mathbb{R}^{+}$. The conical singularity is located at $z_{i}=0, i=1, \ldots, d$. 
It is also an important fact that $c_{1}(X)=0$ is equivalent to the statement that the sum of the $U(1)$ charges is zero for each $U(1)$ factor. Thus

$$
\sum_{i=1}^{d} Q_{a}^{i}=0 \quad a=1, \ldots, r
$$

This latter fact ensures also that the one-loop beta function is zero. The sigma model is then Calabi-Yau, although note that the metric induced by the Kähler quotient is not Ricci-flat.

\subsection{Delzant's construction: from polytopes to gauged linear sigma-models}

Let us first suppose we have a Delzant polytope $\Delta$ which is the image of some compact symplectic toric manifold $M$ under its associated moment map. One can reconstruct $M$ from $\Delta$ as follows. Let $v_{i} \in \mathbb{Z}^{n}, i=1, \ldots, d$, denote the outward pointing primitive normal vectors to the facets of $\Delta$. For some $\lambda_{i} \in \mathbb{R}$ we may then write

$$
\Delta=\left\{x \in \mathbb{R}^{n} \mid<x, v_{i}>\leq \lambda_{i}, i=1, \ldots, d\right\}
$$

Consider now the linear map $\pi: \mathbb{R}^{d} \rightarrow \mathbb{R}^{n}$ which maps the standard basis vectors $E_{i}$ of $\mathbb{R}^{d}$ to $v_{i}$. Thus $\pi\left(E_{i}\right)=v_{i}$ for each $i=1, \ldots, d$. From the Delzant properties of $\Delta$ one easily sees that this map is surjective. The kernel has dimension $r=d-n$, and defines a corresponding torus $\mathbb{T}^{r} \subset \mathbb{T}^{d}$. Now take $\mathbb{C}^{d}$ with its usual action by $\mathbb{T}^{d}$, and consider the moment map where we take the Fayet-Illiopoulos parameters to be $t_{i}=\lambda_{i}$. From the induced action by $\mathbb{T}^{r} \subset \mathbb{T}^{d}$ above, we get an induced moment map for the $\mathbb{T}^{r}$ action. One may now take the symplectic reduction $\mathbb{C}^{d} / / \mathbb{T}^{r}$, which is a symplectic manifold of complex dimension $d-r=d-(d-n)=n$. Moreover, this quotient also inherits an action of $\mathbb{T}^{n}=\mathbb{T}^{d} / \mathbb{T}^{r}$ from that of $\mathbb{C}^{d}$ and is thus toric. In fact, it is not difficult to see that the image of $\mathbb{C}^{d} / / \mathbb{T}^{r}$ under its moment map, associated to $\mathbb{T}^{n}$, is just $\Delta$. This is Delzant's construction [21].

As a completely trivial example, consider the two-sphere $S^{2}$ with canonical $U(1)$ action which rotates about the equator. The image of the moment map is just a line segment, with length proportional to the volume of the two-sphere. The outward pointing normal one-vectors are $v_{1}=1, v_{2}=-1$. The kernel of the map $\pi: E_{i} \mapsto v_{i}$ is thus $(1,1)$, whence we see that $S^{2}$ is the symplectic reduction of $\mathbb{C}^{2}$ by $U(1)$ with charges $(1,1)$ - the $U(1)$ quotient is just the Hopf map $S^{3} \rightarrow S^{2}$. 
There is a corresponding construction for compact symplectic toric orbifolds, which is a generalisation that takes into account that the normals may no longer form a $\mathbb{Z}_{-}^{-}$ basis for $\mathbb{Z}^{n}$. This introduces finite subgroups $\Gamma$ which become local orbifold groups in the symplectic quotient 38 .

\section{A Delzant construction for cones}

Recently a Delzant theorem has been proven for symplectic toric cones [20]. The language used is largely that of contact geometry - recall that a metric cone over a contact manifold is precisely a symplectic cone, and vice versa. The essential point is that the convex rational polyhedral cone one starts with must be good. This ensures that the symplectic quotient is smooth.

Since the moment cones $\mu\left(C\left(Y^{p, q}\right)\right)$ are all good cones, we may apply the theorem of 20]: one simply applies Delzant's construction, as in the compact case, and sets all the Fayet-Iliopoulos parameters to zero. Thus recall that the outward pointing primitive normal vectors were found to be

$$
v_{1}=[1,0,0], \quad v_{2}=[1,-2,-l], \quad v_{3}=[1,-1,-p], \quad v_{4}=[1,-1,0] .
$$

By inspection the kernel is $(p, p,-l,-k)$. Thus the Delzant theorem for cones gives

- $U(1)$ gauged linear sigma-model on $\mathbb{C}^{4}$ with charge vector $Q=(p, p,-l,-k)$.

As a preliminary check this this is indeed correct, notice that the charges sum to zero: $p+p-l-k=0$, since $k=p+q, l=p-q$. It follows that the vacuum manifold $X$ of this gauged linear sigma model is topologically Calabi-Yau, $c_{1}(X)=0$, just as expected. Moreover, by turning on the Fayet-Iliopoulos parameter $t$ for the $U(1)$ gauge field we will obtain orbifold resolutions of the cone.

As interesting degenerate cases, consider $p=1, q=0$. This is the (resolved) conifold, which recall is the gauged linear sigma model on $\mathbb{C}^{4}$ with charges $Q=$ $(1,1,-1,-1)$. Another important case is $p=q=1$. This yields $\mathbb{C}$ times the linear sigma model on $\mathbb{C}^{3}$ with charges $(1,1,-2)$. The latter is $\mathcal{O}_{\mathbb{C P}^{1}}(-2)$. Taking $t=$ 0 shrinks the $\mathbb{C P}^{1}$ to zero size, yielding the orbifold $\mathbb{C}^{2} / \mathbb{Z}_{2}$, which is also the $A_{1}$ singularity. Thus the cone is $\mathbb{C} \times\left(\mathbb{C}^{2} / \mathbb{Z}_{2}\right)$. This has $\mathcal{N}=2$ rather than $\mathcal{N}=1$ supersymmetry. The horizons of these two spaces are thus $T^{1,1}$ and $S^{5} / \mathbb{Z}_{2}$.

If one formally takes $p=q \neq 1$ and $q=0$, one obtains $\mathbb{Z}_{p}$ quotients of the cases above. In particular these will correspond to orbifolds $\left(\mathbb{C}^{2} / \mathbb{Z}_{2} \times \mathbb{C}\right) / \mathbb{Z}_{p}$ and 
(conifold) $/ \mathbb{Z}_{p}$ respectively. It is interesting to note that these are consistent with the limiting volumes (2.14), although the metrics $Y^{p, q}$ are not valid in these limits.

We can now use the results of [40] to perform further non-trivial checks. According to Theorem 1.1 of Ref. [40] we have the following general topological facts about the base $Y$ of the symplectic toric cone $C(Y)$ we began with (provided it is of Reeb type):

- $\pi_{1}(Y) \cong \mathbb{Z}^{n} /<v_{i}>$, is a finite abelian group. Recall that $n=\operatorname{dim}\left(\mathbb{T}^{n}\right)$ is the complex dimension of the cone $C(Y)$.

- $\pi_{2}(Y)$ is a free abelian group of rank $d-n$, where $d$ is the number of facets of the moment cone.

We may now verify that these are indeed true for our examples $Y^{p, q}$ and their moment cones. In particular, for our polyhedral cones recall that the $\left\{v_{i}\right\}$ spanned $\mathbb{Z}^{3}$ over $\mathbb{Z}$, and thus $\pi_{1}\left(Y^{p, q}\right)$ is trivial, in agreement with the fact that $Y^{p, q} \cong S^{2} \times S^{3}$ for all $p, q$. Moreover, we may now relax the condition that $\operatorname{hcf}(p, q)=1$. From the Gysin sequence for the $U(1)$ fibration corresponding to $\partial / \partial \gamma$, as in the appendix of [6], one sees that $\pi_{1}\left(Y^{p, q}\right) \cong \mathbb{Z}_{h}$ where $h \equiv \operatorname{hcf}(p, q)$. Since now $\operatorname{hcf}(l, p)=\operatorname{hcf}(p, q)=h$ Lerman's theorem says that $\pi_{1}\left(Y^{p, q}\right) \cong \mathbb{Z}_{h}$, in agreement with the Gysin sequence calculation.

For the second point in the theorem, since there are four normals, we also learn that $\pi_{2}\left(Y^{p, q}\right) \cong \mathbb{Z}$, again in perfect agreement with the topology we started with.

\subsection{The topology of the vacuum}

In this subsection we verify that one can recover the topology of, as well as the action of the isometry group on, $Y^{p, q}$ correctly as the boundary, or horizon, of the linear sigma model $(p, p,-l,-k)$. Of course, this is guaranteed by the Delzant theorem of [20]. Nevertheless, it is interesting to analyse the relation explicitly, since this sheds considerable light on the geometry and topology.

Since this "hands on" approach is rather technical, the reader might well omit the remainder of this section. However, we will need the relation (15.9) between vectors on $\mathbb{C}^{4}$ and $Y^{p, q}$ in the next section. This section also constitutes a direct proof of the equivalence of the gauged linear sigma models with the Calabi-Yau cones, without using any theorems. 


\section{A direct analysis of the topology}

The point of this subsection is to show that the Kähler quotient $\mathbb{C}^{4} / / U(1)$ is topologically the same as $C\left(Y^{p, q}\right)$. This is far from obvious, but is nevertheless guaranteed by the general theorems we have used thus far.

At $z_{3}=z_{4}=0$ we have a finite sized $\mathbb{C P}^{1}$, of size $t / p$, where recall that $t$ is the FI parameter. We may thus introduce gauge invariant coordinates $z=z_{1} / z_{2}, z^{\prime}=z_{2} / z_{1}$ which cover the open subsets $U_{2}, U_{1} \subset \mathbb{C P}^{1}$ where $U_{i}=\left\{z_{i} \neq 0, z_{3}=z_{4}=0\right\} \subset \mathbb{C P}^{1}$. On the overlap $U_{2} \cap U_{1}$ we have $z=1 / z^{\prime}$, thus making the Riemann sphere. However, for $p>1$ this $\mathbb{C P}^{1}$ is a locus of $\mathbb{Z}_{p}$ orbifold singularities in the Kähler quotient. Indeed, the subgroup $\mathbb{Z}_{p} \subset U(1)$ stabilises the subspace $\left(z_{1}, z_{2}, 0,0\right)$ of $\mathbb{C}^{4}$. The fact that we have a non-trivial isotropy subgroup means that this will descend to a locus of $\mathbb{Z}_{p}$ orbifold singularities in the quotient space. To analyse this singularity, consider, for example, the subspace given by $z_{1}=0$. Using a gauge transformation we may set $z_{2}$ to be real and positive, which is thus the north pole of the base $\mathbb{C P}^{1}=S^{2}$. The action of $\mathbb{Z}_{p}$ on $\left(z_{3}, z_{4}\right)$ is generated by $\left(z_{3}, z_{4}\right) \rightarrow\left(z_{3} \omega_{p}^{-l}, z_{4} \omega_{p}^{-k}\right)$ where $\omega_{p}=e^{2 \pi i / p}$ generates $\mathbb{Z}_{p}$. Note that this is equivalent to the anti-diagonal action $\left(z_{3}, z_{4}\right) \rightarrow\left(z_{3} \omega_{p}^{q}, z_{4} \omega_{p}^{-q}\right)$. Thus if $U(1)_{A} \subset S U(2) \subset U(2)$ acts on $\mathbb{C}^{2}$ in the usual way, we have that the generator $\omega_{p}$ of $\mathbb{Z}_{p}$ embeds in $U(1)_{A}$ as $\omega_{p}^{q}$. Notice that $\left|z_{2}\right|^{2} \geq t / p$ and that, for fixed $\left|z_{2}\right|^{2}>t / p$ the $D$-term imposes that the coordinates $z_{3}, z_{4}$ define an ellipsoid, which topologically is $S^{3}$ modulo the $\mathbb{Z}_{p}$ action just discussed. Since $q$ is prime to $p$ this is the Lens space $L(1, p)$. At $\left|z_{2}\right|^{2}=t / p$ we have $z_{3}=z_{4}=0$ and the Lens space collapses. Thus the subspace $z_{1}=0$ is a copy of an $A_{p-1}$ singularity. Performing the quotient of the Lens space "at infinity" by $U(1)_{A}$ then gives a two-sphere, the map being the $p$ th power of the anti-Hopf map ${ }^{16}$.

Clearly the same picture holds at all points in $\mathbb{C P}^{1}$, not just at $z_{1}=0$, as $S O(3)$ acts as a symmetry. It follows that we have an $A_{p-1}$ fibration over this $\mathbb{C P}^{1}$, which thus has a boundary which is a Lens space bundle over $\mathbb{C P}^{1}$. In fact such a bundle structure of the metrics $Y^{p, q}$ was already noted in reference [6]. We may then quotient the boundary by $U(1)_{A}$ to obtain a space $\hat{B}$ that will be an $S^{2}$ bundle over the base $\mathbb{C P}^{1}=S^{2}$. To see what this bundle is we may introduce coordinates as follows. Suppose $z_{2} \neq 0$, giving the patch $U_{2}$ on the base $\mathbb{C P}^{1}$ with coordinate $z=z_{1} / z_{2}$. In order to effectively go to the boundary of our space, we may set $l\left|z_{3}\right|^{2}+k\left|z_{4}\right|^{2}=$

\footnotetext{
${ }^{16}$ Note the distinction here with the diagonal subgroup $U(1)_{D}$ of $U(2)$. Quotienting by this is the Hopf map, and moreover since this is a normal subgroup the quotient is also group $U(2) / U(1)_{D} \cong$ $S O(3)$. This $S O(3)$ thus acts naturally on the projected space.
} 
constant $>0$. In particular, we cannot have both $z_{3}$ and $z_{4}$ zero. Suppose then that $z_{3} \neq 0$. We may now introduce the additional coordinate $x_{2}=\bar{z}_{4} / z_{3} z_{2}^{2}$ on the fibre. This is invariant under both the original $U(1)$ action - the key point being that $k+l=2 p-$ as well as $U(1)_{A}$ under which the fields have charges $(0,0,1,-1)$. Similarly over $U_{1}$ we have coordinate $x_{1}=\bar{z}_{4} / z_{3} z_{1}^{2}$. The union of these two subspaces thus describes the bundle $\mathcal{O}_{\mathbb{C P}^{1}}(2)$. However, note that, due to the presence of the $\bar{z}_{4} \mathrm{~s}$, the complex structure here is not inherited from the complex structure of $\mathbb{C}^{4}$ we started with. Since we are only interested in topology and group actions, this fact will not be important for the present discussion. Similarly, for $z_{4} \neq 0$ one has coordinates $w_{2}=z_{3} z_{2}^{2} / \bar{z}_{4}$ and $w_{1}=z_{3} z_{1}^{2} / \bar{z}_{4}$. This describes $\mathcal{O}_{\mathbb{C P}^{1}}(-2)$. The intersection of these subspaces results in the gluing of the two $\mathbb{C}$ fibres together to create a Riemann sphere $S^{2}$ bundle over $\mathbb{C P}^{1}=S^{2}$ - for example, $x_{2}=1 / w_{2}$ on the overlap with $z_{2}, z_{3}, z_{4} \neq 0$. Thus we obtain precisely the same description as the manifold $B$ discussed earlier: $\hat{B} \cong B$.

Topologically, the manifold $\hat{B}$ just described is the same thing as $\mathbb{P}(\mathcal{O} \oplus \mathcal{O}(-2))$ which is the second Hirzebruch surface $\mathbb{F}_{2}$. However, due to the $\bar{z}_{4} \mathrm{~s}$, the complex structure is not that inherited from $\mathbb{C}^{4}$. Indeed, if one replaces $\bar{z}_{4}$ by $z_{4}$ in the above coordinates, one precisely gets $\mathbb{F}_{2}$, as one can see by analysing the linear sigma model for this manifold ${ }^{17}$. The fibre $S^{2}$ is thus perhaps best described as $\overline{\mathbb{P P}}^{1}$. Moreover, as explained in [6], as a real manifold $\mathbb{F}_{2}$ is actually just a product space $S^{2} \times S^{2}$ i.e. the bundle is trivial.

It remains to compute the twisting of $U(1)_{A}$ over this base $B$, which as we have just seen is naturally described as an $S^{2}$ bundle over $S^{2}$ with twist 2 . Over the fibre $S^{2}$, sitting at some point on the base $S^{2}=\mathbb{C P}^{1}$, the twisting of the $U(1)$ is $p$, as is clear from the above discussion since the fibre sphere descended from the Lens space $L(1, p) \cong S^{3} / \mathbb{Z}_{p}$. We now compute the $U(1)$ twisting over the copies of $S^{2}$ at the south and north poles of the fibre $S^{2}$ - these are two sections of the $S^{2}$ bundle. Call them $S_{1}$ and $S_{2}$, respectively, as in $\left[\underline{6}\right.$. These are given by $z_{3}=0, z_{4}=0$, respectively, which give linear sigma models on $\mathbb{C}^{3}$ with weights $(p, p,-k),(p, p,-l)$, respectively. The boundaries of these two spaces are Lens spaces $L(1, k), L(1, l)$. To see this, note that $S^{1} / \mathbb{Z}_{p} \cong S^{1}$. Thus the boundaries are $S^{1}$ bundles over $S^{2}$. The twisting in each case is easily seen to be $k$ and $l$, respectively.

We may now relate this to our earlier discussion. Recall that the canonical generators $C_{1}, C_{2}$ of the second homology of $S^{2} \times S^{2}$ are related to the copies $S_{1}, S_{2}$ of $S^{2}$

\footnotetext{
${ }^{17}$ This is a $U(1)^{2}$ model on $\mathbb{C}^{4}$ with charges $Q_{1}=(1,1,2,0), Q_{2}=(0,0,1,1)$.
} 
at the south and north poles of the fibre $S^{2}$ by

$$
\begin{aligned}
& 2 C_{1}=S_{1}-S_{2} \\
& 2 C_{2}=S_{1}+S_{2} .
\end{aligned}
$$

We have just seen that the twisting over $S_{1}$ and $-S_{2}$ is $k$ and $l$, respectively. This gives the Chern numbers over $C_{1}$ and $C_{2}$ to be $(k+l) / 2=p$, and $(k-l) / 2=q$, respectively. We thus precisely reproduce the topology of $Y^{p, q}$ described in section 2. Moreover, the (not quite effectively acting) isometry group of the Sasaki-Einstein metrics is $S U(2) \times U(1)^{2}$. The Kähler quotient above also has this isometry group this is just the subgroup of $U(4)$ that commutes with the original $U(1)$ action.

\section{Relation between Killing vector fields}

It is also now interesting to examine the codimension two fixed point sets of the linear sigma model $(p, p,-l,-k)$ directly, and compare with our polyhedral cone for $C\left(Y^{p, q}\right)$. Thus we now set $t=0$. The codimension two fixed point sets are easily found: they are at $z_{i}=0$, for each $i=1, \ldots, 4$. Indeed, from our above discussion of the topology of the vacuum, these are precisely cones over the Lens spaces $S^{3} / \mathbb{Z}_{p}$, $S^{3} / \mathbb{Z}_{p}, S^{3} / \mathbb{Z}_{k}, S^{3} / \mathbb{Z}_{l}$, respectively. In terms of $Y^{p, q}$, these are the submanifolds $F_{i}$, $i=1, \ldots, 4$, respectively. In particular, note that $F_{3} / U(1) \cong S_{1}, F_{4} / U(1) \cong S_{2}$. Thus we see explicitly that the topology of the subspaces $\left\{z_{i}=0\right\}$ are the same as $C\left(F_{i}\right)$, respectively.

The relation between the Killing vectors is also easy to make explicit. Let us denote $\partial / \partial \theta_{i}$ as the $U(1)$ that rotates the coordinate $z_{i}$. Thus $\partial / \partial \theta_{i}=0$ defines the codimension two submanifolds $z_{i}=0$. We find

$$
\begin{aligned}
2 \frac{\partial}{\partial \phi} & =\frac{\partial}{\partial \theta_{1}}-\frac{\partial}{\partial \theta_{2}} \\
2 p \frac{\partial}{\partial \psi} & =l \frac{\partial}{\partial \theta_{3}}+k \frac{\partial}{\partial \theta_{4}} \\
p \frac{\partial}{\partial \gamma} & =-\frac{\partial}{\partial \theta_{3}}+\frac{\partial}{\partial \theta_{4}} .
\end{aligned}
$$

These require some explanation. We denote the weights of the $\partial / \partial \theta_{i}$ as a row vector for convenience. Thus consider $(1,-1,0,0)$. For $t>0$ this precisely rotates the subspace $z_{3}=z_{4}=0$, which is a copy of $\mathbb{C P}^{1}$ of size $t / p$, with weight two. Hence we identify this $U(1)$ with $2 \partial / \partial \phi$. Also, by construction, the $\partial / \partial \gamma$ direction is proportional to $(0,0,1,-1)$ which recall we denoted $U(1)_{A}$. However, the orbits of the 
vector $(0,0,-1,1)$ actually wind $p$ times around the circle fibre: recall the projection of this $U(1)$ was the $p$ th power of the anti-Hopf map. Hence this is $p \partial / \partial \gamma$. Finally, note that $\partial / \partial \psi$ rotates the fibre $S^{2}$ with weight one and does not act on the base $S^{2}$. This determines the final vector, as one can see by analysing the action on the coordinates $x_{1}, x_{2}, w_{1}, w_{2}$ introduced above.

To make contact with the normal vectors discussed earlier, one must note that the Killing vector given by $(p, p,-l,-k)$ acts trivially on the vacuum, by construction. Thus $(p,-p, 0,0)$ is equivalent to both $(2 p, 0,-l,-k)$ and $(0,-2 p, l, k)$. Thus we compute

$$
\begin{aligned}
\frac{\partial}{\partial \phi}+\frac{\partial}{\partial \psi} & =\frac{\partial}{\partial \theta_{1}} \\
-\frac{\partial}{\partial \phi}+\frac{\partial}{\partial \psi} & =\frac{\partial}{\partial \theta_{2}} \\
\frac{\partial}{\partial \psi}-\frac{k}{2} \frac{\partial}{\partial \gamma} & =\frac{\partial}{\partial \theta_{3}} \\
\frac{\partial}{\partial \psi}+\frac{l}{2} \frac{\partial}{\partial \gamma} & =\frac{\partial}{\partial \theta_{4}}
\end{aligned}
$$

in perfect agreement with our earlier results: the vectors on the left hand side are precisely the Killing vectors (4.9) which fixed codimension two submanifolds of $Y^{p, q}$. In particular, this means that the polyhedral cones for $C\left(Y^{p, q}\right)$ and the linear sigma model with weights $(p, p,-l,-k)$ are identical, and thus they are completely equivalent as symplectic toric cones i.e. they are equivariantly symplectomorphic. We have shown this directly in this subsection, without appealing to any theorems.

\section{Toric Gorenstein canonical singularities}

In this section we make contact with reference [5] by explaining the relation of the Calabi-Yau gauged linear sigma model $(p, p,-l,-k)$ to so-called toric Gorenstein canonical singularities.

The data required to define a toric Gorenstein canonical singularity of complex dimension $n$ is a convex polygon on $\mathbb{R}^{n-1}$, all of whose vertices have integer coordinates. Given any such polygon one can reconstruct the toric singularity, as well as all of its toric crepant resolutions, as follows. Let $\left\{\mathcal{V}_{i} \mid i=1, \ldots, d\right\}$ denote all vectors in

$\mathbb{R}^{n-1}$ with integer coordinates and with the property that they lie within, or on the boundary of, the polygon. Marking these points gives the toric diagram $\mathcal{D}$. Consider 
now the set of all linear relations among these vectors

$$
\sum_{i=1}^{d} Q_{a}^{i} \mathcal{V}_{i}=0
$$

with integer coefficients $Q_{a}^{i}$ satisfying

$$
\sum_{i=1}^{d} Q_{a}^{i}=0
$$

for each $a=1, \ldots r$, where $a$ labels the set of such linear relations. Clearly $r=d-n$. One now uses the matrix $Q_{a}^{i}$ as the charges of a linear sigma model on $\mathbb{C}^{d}$ with gauge group $U(1)^{r}$. This is essentially a Delzant construction. The Kähler quotient $X=\mathbb{C}^{d} / / U(1)^{r}$ has complex dimension $n=d-r$. Setting all FI parameters to zero gives the toric singularity. Moreover, by turning on the FI parameters one obtains (partial) resolutions of the singularity - special values of the FI parameters will give rise to more singular spaces than the generic values. By including all the interior points $\mathcal{V}_{i}$ of the polygon, we have ensured that the linear sigma model reproduces all the toric crepant resolutions of the singularity. The sizes of the blow-ups are controlled by the FI parameters. However, this is not usually a very economical way of constructing the singularity - the minimal presentation, meaning the smallest possible $d$ and thus least number of chiral superfields, arises by using only the vertices of the polygon ${ }^{18}$.

The toric diagram for the Calabi-Yau cone on $Y^{p, q}$ can be obtained as follows. Recall that the image of the moment map for $C\left(Y^{p, q}\right)$ is a four-faceted polyhedral cone with primitive outward pointing normals

$$
v_{1}=[1,0,0], \quad v_{2}=[1,-2,-l], \quad v_{3}=[1,-1,-p], \quad v_{4}=[1,-1,0] .
$$

Notice that these vectors lie in the plane at $e_{1}=1$. Indeed, the normals belong to a plane in $\mathbb{R}^{2}$ precisely when the linear sigma model is Calabi-Yau. Thus we may project onto the $e_{1}=1$ plane to obtain vectors

$$
[0,0], \quad[-2,-l], \quad[-1,-p], \quad[-1,0] .
$$

We now shift the origin by $[1,0]$ and then make the $S L(2 ; \mathbb{Z})$ transformation

$$
\left(\begin{array}{cc}
l-1 & -1 \\
l & -1
\end{array}\right)
$$

\footnotetext{
${ }^{18}$ If these vectors do not span $\mathbb{Z}^{n-1}$ over $\mathbb{Z}$ one must in addition quotient the Kähler quotient by the finite group $\mathbb{Z}^{n-1} /<\mathcal{V}_{i}>$ to correctly reproduce the singularity - this follows from our general discussion in Section 3.
} 
to obtain vectors

$$
\mathcal{V}_{1}=[l-1, l], \quad \mathcal{V}_{2}=[1,0], \quad \mathcal{V}_{3}=[p, p], \quad \mathcal{V}_{4}=[0,0]
$$

respectively. This is a minimal presentation of the singularity. The pictures below display some examples with low values of $p$. It is interesting to note that the areas

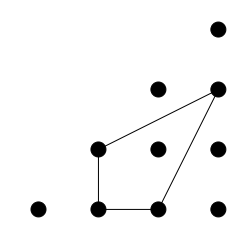

Figure 1: Toric diagram of $Y^{2,1}$ embedded in the orbifold $\mathbb{C}^{3} / \mathbb{Z}_{3} \times \mathbb{Z}_{3}$.

of these polygons are equal to $p$, independently of $q$. Indeed, for fixed $p$, varying $q$ just slides the vertex $\mathcal{V}_{1}$ up and down the hypotenuse of the triangle that defines the orbifold $\mathbb{C}^{3} / \mathbb{Z}_{p+1} \times \mathbb{Z}_{p+1}$. Note that for $(p, q)=(2,1)$ the toric diagram is the same as that for the complex cone (canonical line bundle) over the first del Pezzo surface, as we discuss in detail in the following section.
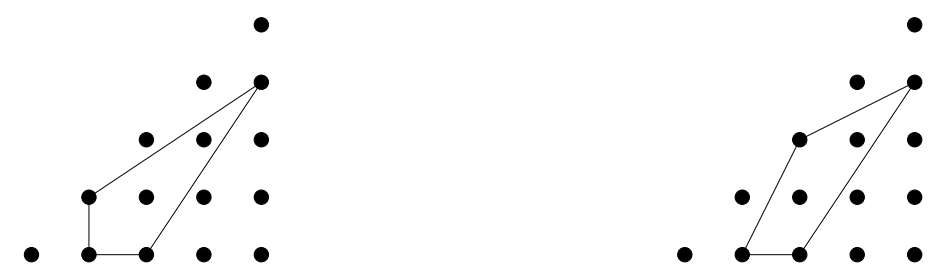

Figure 2: Toric diagrams of $Y^{3,2}$ and $Y^{3,1}$ embedded in the orbifold $\mathbb{C}^{3} / \mathbb{Z}_{4} \times \mathbb{Z}_{4}$.

Let us also remark that the number of points inside the polygon is precisely $p-1$. Each point corresponds to a normal vector to a plane in $\mathbb{R}^{3}$. The total number of Fayet-Iliopoulos parameters (Kähler parameters) is $(4-3)+p-1=p$, and by varying these one moves the planes in their normal directions so that they no longer intersect the origin. By assigning generic values one completely resolves the conical singularity. Indeed, these parameters roughly control the size of $\mathbb{C P}^{1} \mathrm{~s}$. We thus learn that the Calabi-Yau cone, where all FI parameters are set to zero, has $p$ collapsed two-spheres. 
Turning on the FI parameter $t>0$ in the linear sigma model $(p, p,-l,-k)$ partially resolves the singularity to an $A_{p-1}$ singularity fibred over $\mathbb{C P}^{1}$, as discussed in the last section. Indeed, an $A_{p-1}$ singularity can be completely resolved by blowing up $(p-1)$ two-spheres - the metric is the $p$-centered Gibbons-Hawking metric. There are precisely $(p-1)$ FI parameters, giving $1+(p-1)=p$ in total.

\section{The complex cone over $\mathbb{F}_{1}$}

As noted above, the toric diagram we have found for $Y^{2,1}$ is the same as that for the complex cone over the first del Pezzo surface. We will refer to the latter as $\mathbb{F}_{1}$ and its complex Calabi-Yau cone as $C_{\mathbb{C}}\left(\mathbb{F}_{1}\right)$. Here we elaborate on this point. In particular, it follows that we will inherit a metric on $\mathbb{F}_{1}$ from that on $Y^{2,1}$, and we will write this down explicitly. Of course this metric will not be Kähler-Einstein.

First we will use the toric data we have to deduce the Killing vector field on $Y^{2,1}$ corresponding to the complex cone direction. Adapting the metric to this direction, we shall indeed find a smooth metric on $\mathbb{F}_{1}$.

We label the five vertices of the toric diagram, including the blow-up mode corresponding to the interior point, as

$$
\mathcal{V}_{1}=[0,1], \quad \mathcal{V}_{2}=[1,0], \quad \mathcal{V}_{3}=[2,2], \quad \mathcal{V}_{4}=[0,0], \quad \mathcal{V}_{5}=[1,1]
$$

The last vector $\mathcal{V}_{5}$ is the additional blow-up vertex. A possible basis for the two charge vectors is given by

$$
\begin{aligned}
& Q_{1}=(1,1,0,-1,-1) \\
& Q_{2}=(0,0,1,1,-2) .
\end{aligned}
$$

We thus obtain a gauged linear sigma model on $\mathbb{C}^{5}$ with $U(1)^{2}$ gauge group. Let us for the moment drop the last entry in these vectors. This gives a gauged linear sigma model on $\mathbb{C}^{4}$ with weights

$$
\begin{aligned}
& \hat{Q}_{1}=(1,1,0,-1) \\
& \hat{Q}_{2}=(0,0,1,1) .
\end{aligned}
$$

Let us take each quotient in turn. The first quotient yields $\mathbb{C} \times\left[\mathcal{O}_{\mathbb{C P}^{1}}(-1)\right]$, since $(1,1,-1)$ is precisely $\mathcal{O}_{\mathbb{C P}^{1}}(-1)$. The former may also be regarded as $\mathcal{O}_{\mathbb{C P}^{1}}(0) \oplus$ $\mathcal{O}_{\mathbb{C P}^{1}}(-1)$. The second row then projectivises this $\mathbb{C}^{2}=\mathbb{C} \oplus \mathbb{C}$ bundle. This means 
one quotients each $\mathbb{C}^{2}$ fibre by the Hopf map $\mathbb{C}^{2} \backslash\{0\} \rightarrow \mathbb{C P}^{1}$. The resulting space is the first Hirzebruch surface

$$
\mathbb{F}_{1}=\mathbb{P}\left(\mathcal{O}_{\mathbb{C P}^{1}}(0) \oplus \mathcal{O}_{\mathbb{C P}^{1}}(-1)\right)
$$

This is also the same thing as $\mathbb{C P}^{2}$ blown up at a point ${ }^{19}$. Indeed, $\mathbb{C P}^{2}$ may be obtained by taking $\mathcal{O}(1) \rightarrow \mathbb{C P}^{1}$ and gluing to its boundary, which is topologically $S^{3}$, a ball in $\mathbb{C}^{2}$. Blowing up the origin in $\mathbb{C}^{2}$ replaces it by a $\mathbb{C P}^{1}$, which has local geometry $\mathcal{O}_{\mathbb{C P}^{1}}(-1)$. Equivalently one can describe this blowing up process as taking a connected sum with $\mathbb{C P}^{2}$ with reversed orientation: $\mathbb{C P}^{2} \#-\mathbb{C P}^{2}$. We now have two copies of $\mathbb{C P}^{1}$ in the resulting space. In fact it is easy to see that these are two sections of $\mathbb{F}_{1}$ - this is precisely analogous to the topological construction of $B$. Note however that $w_{2}\left(\mathbb{F}_{1}\right) \neq 0$ and thus this is not a spin manifold.

Adding back the fifth entry to the charge vectors (7.3) to give (17.2) then describes the canonical bundle over $\mathbb{F}_{1}$ - the charges sum to zero, meaning that the vacuum $X$ (Kähler quotient) is topologically Calabi-Yau, $c_{1}(X)=0$. This identifies the canonical bundle, or complex cone, over $\mathbb{F}_{1}$.

Consider now taking a different linear combination of charge vectors, corresponding to a change of basis for the $\mathbb{T}^{2}$ action. In particular, using an $S L(2 ; \mathbb{Z})$ transformation we may take

$$
\begin{aligned}
& Q_{1}^{\prime}=(2,2,-1,-3,0) \\
& Q_{2}^{\prime}=(1,1,0,-1,-1) .
\end{aligned}
$$

The first set of weights of course gives the gauged linear sigma model on $\mathbb{C}^{4}$ given by $(2,2,-1,-3)=(p, p,-l,-k)$, together with a factor of $\mathbb{C}$. We may now effectively gauge away the second $U(1)$. Indeed, this means

$$
\frac{\partial}{\partial \theta_{5}}=-\frac{\partial}{\partial \theta_{4}}+\frac{\partial}{\partial \theta_{1}}+\frac{\partial}{\partial \theta_{2}}=\frac{\partial}{\partial \psi}-\frac{1}{2} \frac{\partial}{\partial \gamma} .
$$

acting on the linear sigma model $(2,2,-1,-3)$ on $\mathbb{C}^{4}$, and $Y^{2,1}$, respectively. Here we have used the relations (15.9). Note that $\partial / \partial \theta_{5}$ precisely rotates the complex line fibre over $\mathbb{F}_{1}$. One can check explicitly that this Killing vector field on $Y^{2,1}$ is nowhere-vanishing. Indeed, its norm-squared is computed to be

$$
\left|\frac{\partial}{\partial \theta_{5}}\right|^{2}=F(y) \equiv \frac{q(y)}{9}+w(y)\left(f(y)-\frac{1}{2} \ell\right)^{2}
$$

\footnotetext{
${ }^{19}$ In the toric language, there is a nice way to understand this. In fact, it's straightforward to compute the Delzant polytope for $\mathbb{C P}^{2}$ : this is an isosceles rectangular triangle. A toric blow-up is obtained by simply chopping off a vertex to give a rectangular trapezoid.
} 
which is strictly positive. Here

$$
f(y)=\frac{a-2 y+y^{2}}{6\left(a-y^{2}\right)}
$$

is the function appearing in the local one-form $A$. Of course in this particular case $a$ and $\ell$ take specific values. One finds

$$
\begin{aligned}
a & =\frac{1}{2}\left(1-\frac{\sqrt{13}}{16}\right) & \ell & =\frac{1}{2 \sqrt{13}-5} \\
y_{1} & =\frac{1}{8}(1-\sqrt{13}) & y_{2} & =\frac{1}{8}(7-\sqrt{13}) .
\end{aligned}
$$

Let us summarise the situation. We have found that the metric $Y^{2,1}$ is an explicit irregular Sasaki-Einstein metric on the horizon of the complex cone $C_{\mathbb{C}}\left(\mathbb{F}_{1}\right)$ over $\mathbb{F}_{1}$, where the Killing vector field (7.6) rotates the complex cone direction. Crucially this is not the Reeb vector, whose generic orbits in fact don't close. The quotient of the metric (2.4) by the $U(1)$ action generated by (7.6) should be a metric on $\mathbb{F}_{1}$. We will now explicitly compute this metric and verify that it is indeed a smooth metric on $\mathbb{F}_{1}$.

In order to perform the $U(1)$ quotient of $Y^{2,1}$, it is useful to first rewrite the metric adapted to the Killing vector field $\partial / \partial \theta_{5}$. Thus, let us change coordinates:

$$
\psi=\theta_{5}, \quad \gamma=-\Omega / 2-\theta_{5} / 2 .
$$

It is then straightforward to compute the following expression for the metric

$$
\begin{aligned}
\mathrm{d} s^{2}=\frac{1-y}{6} & \left(\mathrm{~d} \theta^{2}+\sin ^{2} \theta \mathrm{d} \phi^{2}\right)+\frac{1}{w(y) q(y)} \mathrm{d} y^{2}+\frac{w(y) q(y) \ell^{2}}{36 F(y)}(\mathrm{d} \Omega+\cos \theta \mathrm{d} \phi)^{2} \\
& +F(y)\left[\mathrm{d} \theta_{5}-C\right]^{2}
\end{aligned}
$$

where we have defined

$$
C=\frac{1}{F(y)}\left[w(y)\left(f(y)-\frac{\ell}{2}\right) \frac{\ell}{2} \mathrm{~d} \Omega+\left(\frac{q(y)}{9}+w(y) f(y)\left(f(y)-\frac{\ell}{2}\right)\right) \cos \theta \mathrm{d} \phi\right] .
$$

The quotient by $\partial / \partial \theta_{5}$ now simply gives the metric in the first line of (7.11), which again looks like a bundle over a base two-sphere. Let us now analyze regularity of this metric.

First, notice that all the functions are positive semi-definite. So, as usual, one has to worry only about the smoothness conditions where the function $q(y)$ vanishes, and 
then check that the resulting periodicities give a well-defined bundle-metric. Near such a zero $y_{i}$, the "fibre metric", i.e. the metric at fixed $\theta, \phi$, takes the form

$$
\mathrm{d} s^{2}(\text { fibre }) \approx \frac{1}{12\left|y_{i}\right|\left|y-y_{i}\right|} \mathrm{d} y^{2}+\frac{\left|y_{i}\right|\left|y-y_{i}\right| \ell^{2}}{3 F\left(y_{i}\right)} \mathrm{d} \Omega^{2} .
$$

Now, crucially, the following relations are true for any $(p, q)$ :

$$
F\left(y_{1}\right)=(k-1)^{2} \ell^{2} y_{1}^{2} \quad F\left(y_{2}\right)=(l+1)^{2} \ell^{2} y_{2}^{2} .
$$

Introducing $R=2\left|y-y_{i}\right|^{1 / 2}$ we find that for $(k, l)=(3,1)$ - and only for these values - the metric approaches

$$
\mathrm{d} s^{2}(\text { fibre }) \approx \frac{1}{12\left|y_{i}\right|}\left(\mathrm{d} R^{2}+\frac{1}{4} R^{2} \mathrm{~d} \Omega^{2}\right)
$$

near the two zeros. We therefore obtain a smooth metric on $\mathbb{R}^{2}$ in this neighbourhood if and only if $\Omega$ has period $4 \pi$. Indeed, one can see that this is the induced period for $\Omega$ from the metric on $Y^{2,1}$ by examining the coordinate transformation (17.10): since $\psi, \gamma$ and $\theta_{5}$ all have period $2 \pi$ one can calculate the period of $\Omega$ from the Jabobian of the coordinate transformation (7.10), which is $-1 / 2$. This indeed means that $\Omega \sim \Omega+4 \pi$ and moreover with this period we have that for fixed $y, y_{1}<y<y_{2}$, the resulting space is a squashed $S^{3}$. These are then the generic orbits under the action of the isometry group $U(2)$ on this manifold. We thus obtain an $S^{2}$ bundle over $S^{2}$ with twist one, which is topologically $\mathbb{F}_{1}$, just as expected.

Let us now label the two sections of $\mathbb{F}_{1}$ at $y=y_{1}, y=y_{2}$ as $H, E$ respectively. These are the hyperplane class and exceptional divisor of del Pezzo one, respectively. It is a simple exercise to compute the Chern numbers of the $U(1)$ principle bundle, with coordinate $\theta_{5}$, over these:

$$
\int_{H} \frac{\mathrm{d} C}{2 \pi}=3 \quad \int_{E} \frac{\mathrm{d} C}{2 \pi}=1
$$

where, as ever, we have to use the cubic (2.7) and, in this particular case, $k=3, l=1$. Equations (17.16) give precisely the Chern numbers required so that the complex cone (or complex line bundle) defined by the $U(1)$ bundle associated to $\theta_{5}$ is indeed CalabiYau. To see this, notice that the normal bundles of the two $\mathbb{C P}^{1} \mathrm{~s}$ corresponding to $H, E$ inside $\mathbb{F}_{1}$ are topologically $\mathcal{O}_{\mathbb{C P}^{1}}(1)$ and $\mathcal{O}_{\mathbb{C P}^{1}}(-1)$, respectively, as is clear from our discussion of $\mathbb{F}_{1}$ above. Thus $c_{1}\left(\mathbb{F}_{1}\right)$ restricted to the two cycles gives $1+2=3$ and $-1+2=1$, respectively, where $2=c_{1}\left(T S^{2}\right)$. The Chern numbers above for $-\mathrm{d} C$ 
precisely cancel these in the total space of the associated complex line bundle, thus giving a Calabi-Yau manifold.

As shown at the end of Section 2.2, the cones over the $U(1)$ bundles over $H$ and $E$ (which are the submanifolds $y=y_{1}, y=y_{2}$ ) are divisors in the Calabi-Yau cone. Equivalently, the complex cones over the submanifolds $H$ and $E$ are divisors. Indeed, we already noted above the normal bundles to these submanifolds inside $\mathbb{F}_{1}$, which translate into self-intersection numbers $H \cdot H=1, E \cdot E=-1$.

One can check that the metric on $\mathbb{F}_{1}$ is not Einstein. Thus, in particular it is not diffeomorphic to the Page metric on $\mathbb{F}_{1}$ [41], although it is rather similar in form.

\section{New non-trivial AdS/CFT predictions}

In this final section we discuss features of the gauge theory duals of the SasakiEinstein manifolds $Y^{p, q}$, focusing in particular on $Y^{2,1}$ since a candidate dual is already known. In particular we may compare our geometrical results to the $a^{-}$ maximisation calculation ${ }^{20}$ presented in [14]. We find complete agreement with this field theory calculation, both for the central charge and for the $S U(2)_{F}$ singlet baryons of the theory.

Let us first remark that, given a toric Gorenstein canonical singularity, an algorithm for constructing ${ }^{21}$ a quiver gauge theory that has the singularity as its Higgs branch has been developed in [11, 42] and subsequent works by these authors. This relies on the fact that any such singularity may be obtained by partial resolution of the orbifold $\mathbb{C}^{3} / \mathbb{Z}_{p+1} \times \mathbb{Z}_{p+1}$, and the field theory for the latter is known. In practise the algorithm requires a computer, even for relatively small $p$. However, the simple analytic expressions found in this paper suggest that all theories can be treated simultaneously. Indeed it is tempting to speculate that some members of the family could be related by deformations or connected via RG-flows. In particular, we can anticipate that, at fixed $p$, the parameter $q$ will govern the matter content and superpotential of an $S U(N)^{2 p}$ quiver. Recall also that at fixed $p$, the central charge $a$ is a monotonic function of $q$ which is bounded between the values corresponding to

\footnotetext{
${ }^{20}$ Note that in [14] the central charge of the $d P_{2}$ quiver gauge theory is also calculated, and found to be quadratic irrational.

${ }^{21}$ Note that, in earlier work, extending that of $[\underline{3}$, the quiver gauge theories associated to some toric singularities were worked out in [43, 44, 45] without using these algorithms.
} 
$T^{1,1} / \mathbb{Z}_{p}$ and $\left(S^{5} / \mathbb{Z}_{2}\right) / \mathbb{Z}_{p}$

$$
a\left(T^{1,1} / \mathbb{Z}_{p}\right)<a\left(Y^{p, q}\right)<a\left(S^{5} / \mathbb{Z}_{2} \times \mathbb{Z}_{p}\right)
$$

suggesting that the different $q$-theories might all be related to the same "parent" orbifold model. However, we will not pursue this direction any further in the present paper. Instead, we focus on $Y^{2,1}$ where the dual quiver theory is already known. This instance already captures many of the essentially new features of these AdS/CFT duals.

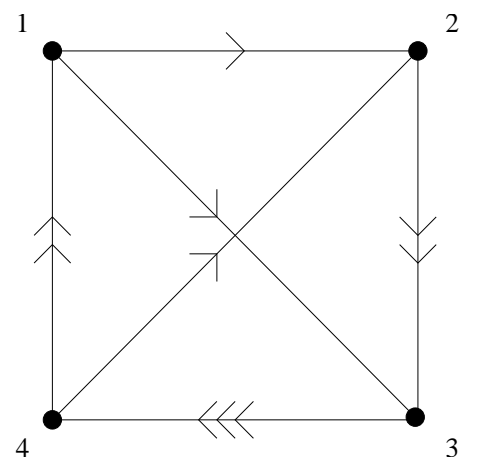

Figure 3: Quiver diagram associated to the complex cone over $d P_{1}$.

A quiver gauge theory for $d P_{1} \cong \mathbb{F}_{1}$ was obtained ${ }^{22}$ in [1] and is presented in Figure 3. Let us briefly recall the notation of these diagrams. The nodes of the diagram represent different gauge group factors $U(N)$. Thus the gauge group for the theory is $U(N)^{4}$. An arrow from node $i$ to node $j$ represents a bifundamental field in the representation $\overline{\mathbf{N}} \otimes \mathbf{N}$, where the first factor denotes the anti-fundamental representation of the $i$ th gauge group, and the second factor denotes the fundamental representation of the $j$ th gauge group. We denote these fields as $X_{i j}$. Thus the quiver diagram encodes the field content of the theory. One must also specify the superpotential. This is given by 42 :

$$
W=\epsilon_{\alpha \beta} \operatorname{tr}\left[X_{34}^{\alpha} X_{41}^{\beta} X_{13}\right]-\epsilon_{\alpha \beta} \operatorname{tr}\left[X_{34}^{\alpha} X_{23}^{\beta} X_{42}\right]+\epsilon_{\alpha \beta} \operatorname{tr}\left[X_{12} X_{34}^{3} X_{41}^{\alpha} X_{23}^{\beta}\right]
$$

where $\epsilon_{\alpha \beta} \in\{ \pm 1\}$ and $\alpha, \beta \in\{1,2\}$ are indices of the non-abelian flavor symmetry group $S U(2)_{F}$. Note that each term comes from a closed loop in the quiver. This

\footnotetext{
${ }^{22}$ In this section we denote the first del Pezzo surface by $d P_{1}$.
} 
allows one to construct gauge-invariant monomials, which may then appear in the superpotential.

One is then particularly interested in the Higgs branch of such a theory. This arises by considering $U(N) \rightarrow U(1)^{N}$ for each gauge group factor. One effectively considers the case $N=1$ so that the gauge theory is an abelian theory - the case $N>1$ will simply be given by $N$ copies of the $N=1$ case. The fields $X_{i j}$ have various charges under the $U(1)^{4}$ gauge group. Setting the $D$-terms of the gauge theory to zero and dividing by the gauge group is, as we have discussed already in a different context in this paper, a Kähler quotient construction, and the result is a toric variety (an overall $U(1)$ decouples and is physically the centre of mass $U(1)$ of the D3-branes). However, to get the vacuum of the theory one must also set the $F$-terms to zero, which means extremising the superpotential: $\mathrm{d} W=0$. This gives a system of relations among the linear sigma model fields, which define hypersurfaces in the toric variety - the intersection of these define the Higgs branch of the theory, which is part of the moduli space of vacua. One can also get to this result by computing all invariant monomials in the fields, and then finding all relations among them, including those relations given by $\mathrm{d} W=0$. The slightly non-trivial fact is that this is indeed the complex cone over the first del Pezzo surface. We will not review this here, but instead refer the reader to the literature for details (see e.g. [23]). If the quiver gauge theory above is interpreted as living on a D3-brane, then this moduli space should be the geometry seen by the brane. For $N>1$ one has $N$ D3-branes in their Higgs phase, which is why one obtains $N$ copies of the above moduli space.

Let us now recall the flavour and $\mathrm{R}$-symmetries of the theory. The superpotential above is manifestly invariant under the non-abelian flavour group $S U(2)_{F}$, for which the $\alpha, \beta$ indices form a doublet. Crucially, there is also a non-anomalous $U(1) \times U(1)$ abelian flavour symmetry which is preserved in the IR. Taking this into account, the $a$-maximisation calculation applied to this theory [14] then gives the exact $\mathrm{R}$-charges in the IR. For the sake of clarity, these are listed ${ }^{23}$ in Table 1 .

Recall that, as proposed in [9], the R-symmetry mixes with the abelian flavour symmetries maximising, among all such admissible $\mathrm{R}$-symmetries, a certain combination of ' $t$ Hooft anomalies. The value of this combination of anomalies at the critical point is the exact central charge of the theory in the infra-red, and is given

\footnotetext{
${ }^{23}$ We thank the authors of [14 for communicating the results of their calculation prior to publication.
} 


\begin{tabular}{|cc|}
\hline$X_{i j}$ & $R_{\text {exact }}$ \\
\hline$X_{34}^{\alpha}$ & $\frac{1}{3}(-1+\sqrt{13})$ \\
$X_{34}^{3}$ & $-3+\sqrt{13}$ \\
$X_{41}^{\alpha}$ & $\frac{4}{3}(4-\sqrt{13})$ \\
$X_{23}^{\alpha}$ & $\frac{4}{3}(4-\sqrt{13})$ \\
$X_{12}$ & $\frac{1}{3}(-17+5 \sqrt{13})$ \\
$X_{13}$ & $-3+\sqrt{13}$ \\
$X_{42}$ & $-3+\sqrt{13}$ \\
\hline
\end{tabular}

Table 1: Exact R-charges computed from $a$-maximisation [14].

by the formula

$$
a=\frac{3}{32}\left(3 \operatorname{Tr} R^{3}-\operatorname{Tr} R\right) .
$$

Substituting the values for the R-charges from Table 1 into (8.3), and comparing with (1.2) one finds a corresponding volume

$$
\frac{13 \sqrt{13}+46}{12 \cdot 27} \pi^{3}
$$

which precisely agrees with the volume of $Y^{2,1}(2.13)$ on setting $p=2, q=1$.

Let us finally consider the baryons of the gauge theory. Recall that baryonic operators $B$ of the gauge theory are dual to D3-branes wrapping supersymmetric cycles $\Sigma$ in the geometry. Their $\mathrm{R}$-charges are related to the volumes of these supersymmetric cycles according to the general formula ${ }^{24}[25]$

$$
R[B]=\frac{2}{3} \cdot\left(\frac{\pi}{2 \operatorname{vol}(Y)}\right) \cdot \operatorname{vol}(\Sigma) .
$$

Recall we have shown in Section 2 that for each manifold $Y^{p, q}$ there are two supersymmetric 3-cycles, which are topologically Lens spaces $\Sigma_{1}=S^{3} / \mathbb{Z}_{p+q}$ and $\Sigma_{2}=S^{3} / Z_{p-q}$. We therefore expect that in each case there will be two types of baryonic operators $B_{1}, B_{2}$ associated to them. Substituting for the volume (2.13) we can write down the general formula for the $\mathrm{R}$-charges of the corresponding baryons in the $Y^{p, q}$ theory. These are given by the unlikely formulae:

$$
\begin{aligned}
& R\left[B_{1}\right]=\frac{1}{3 q^{2}}\left[-4 p^{2}+2 p q+3 q^{2}+(2 p-q) \sqrt{4 p^{2}-3 q^{2}}\right] \\
& R\left[B_{2}\right]=\frac{1}{3 q^{2}}\left[-4 p^{2}-2 p q+3 q^{2}+(2 p+q) \sqrt{4 p^{2}-3 q^{2}}\right] .
\end{aligned}
$$

\footnotetext{
${ }^{24}$ We suppress the overall factors of $N$.
} 
Note that they are interchanged by changing the sign of $q$. Setting $p=2, q=1$ the formulae give

$$
R\left[B_{1}\right]=-3+\sqrt{13}, \quad R\left[B_{2}\right]=\frac{1}{3}(-17+5 \sqrt{13}) .
$$

These agree precisely with two of the four different R-charges listed in Table 1

\section{Acknowledgments}

We would like to thank M. Bertolini, F. Bigazzi, A. Hanany, K. Intriligator, E. Lerman, C. Vafa, D. Waldram, B. Wecht, and S.-T. Yau for discussions and e-mail correspondence. In particular we would like to thank E. Lerman for comments on a draft version of this paper. We are also grateful to the authors of [14] for earlier collaboration on related material, and especially for communicating their $a$-maximisation calculation. DM would like to thank the 2004 Simons Workshop on Mathematics and Physics, for hospitality at initial stages of this work. Part of this work was carried out whilst both authors were postdoctoral fellows at Imperial College, London. In particular DM was funded by a Marie Curie Individual Fellowship under contract number HPMF-CT-2002-01539, while JFS was supported by an EPSRC mathematics fellowship. At present JFS is supported by NSF grants DMS-0244464, DMS-0074329 and DMS-9803347.

\section{A The conifold}

In this appendix we compute the moment cone, gauged linear sigma model and toric diagram for the conifold, $C\left(T^{1,1}\right)$. Of course, many of these results are well-known in the physics literature - we include the discussion only as a simple illustration of the systematic techniques used in this paper, in the context of an example familiar to many physicists.

The homogeneous Sasaki-Einstein metric on $S^{2} \times S^{3}$ is usually referred to as $T^{1,1}$. The metric is particularly simple [4]:

$$
\mathrm{d} s^{2}=\frac{1}{6}\left(\mathrm{~d} \theta_{1}^{2}+\sin ^{2} \theta_{1} \mathrm{~d} \phi_{1}^{2}+\mathrm{d} \theta_{2}^{2}+\sin ^{2} \theta_{2} \mathrm{~d} \phi_{2}^{2}\right)+\frac{1}{9}\left(\mathrm{~d} \psi+\cos \theta_{1} \mathrm{~d} \phi_{1}+\cos \theta_{2} \mathrm{~d} \phi_{2}\right)^{2} .
$$

Here $\theta_{i}, \phi_{i}, i=1,2$, are usual polar and axial coordinates on two round two-spheres, and $\psi$ is a coordinate on a principle $U(1)$ bundle over $S^{2} \times S^{2}$. Here $\psi$ has period 
$4 \pi$ so that the Chern numbers over the two two-spheres are both equal to one ${ }^{25}$. In particular, $3 \partial / \partial \psi$ is the Reeb vector so that this is a regular Sasaki-Einstein manifold - the base Kähler-Einstein manifold is just $\mathbb{C P}^{1} \times \mathbb{C P}^{1}$.

The symplectic form on the metric cone is

$$
\omega=\frac{1}{6} r^{2}\left(\sin \theta_{1} \mathrm{~d} \theta_{1} \wedge \mathrm{d} \phi_{1}+\sin \theta_{2} \mathrm{~d} \theta_{2} \wedge \mathrm{d} \phi_{2}\right)-\frac{1}{3} r \mathrm{~d} r \wedge\left(\mathrm{d} \psi+\cos \theta_{1} \mathrm{~d} \phi_{1}+\cos \theta_{2} \mathrm{~d} \phi_{2}\right) .
$$

Clearly we have three commuting Hamiltonian $U(1) \mathrm{s}$ generated by $\partial / \partial \phi_{i}, i=1,2$, and $\partial / \partial \psi$. As in the main text, one must be careful to ensure that one picks a basis for an effectively acting $\mathbb{T}^{3}$ action when computing the moment map. If one fixes $\theta_{1}, \phi_{1}$ on the first two-sphere, one obtains a copy of $S^{3}$, written as a principle $U(1)$ bundle over the second two-sphere. The effectively acting isometry group on this squashed $S^{3}$ is $U(2)$, as discussed in the main text. Defining $2 \nu=\psi$, so that $\nu$ has canonical period $2 \pi$, one can therefore take the following basis for the $\mathbb{T}^{3}$ action:

$$
\begin{aligned}
& e_{1}=\frac{\partial}{\partial \phi_{1}}+\frac{1}{2} \frac{\partial}{\partial \nu} \\
& e_{2}=\frac{\partial}{\partial \phi_{2}}+\frac{1}{2} \frac{\partial}{\partial \nu} \\
& e_{3}=\frac{\partial}{\partial \nu} .
\end{aligned}
$$

The corresponding moment map, homogeneous under rescaling of the cone, is now easily computed to be

$$
\vec{\mu}=\left(\frac{1}{6} r^{2}\left(\cos \theta_{1}+1\right), \frac{1}{6} r^{2}\left(\cos \theta_{2}+1\right), \frac{1}{3} r^{2}\right) .
$$

The image of the moment map $\mu: C\left(T^{1,1}\right) \rightarrow \mathbb{R}^{3}$ is a convex rational polyhedral cone generated by the four edge vectors:

$$
\begin{aligned}
\vec{\mu}(N N) & =\frac{1}{3}(1,1,1) \\
\vec{\mu}(N S) & =\frac{1}{3}(1,0,1) \\
\vec{\mu}(S N) & =\frac{1}{3}(0,1,1) \\
\vec{\mu}(S S) & =\frac{1}{3}(0,0,1) .
\end{aligned}
$$

That is, the subspaces over which a $\mathbb{T}^{2}$ collapses are precisely the four subspaces $N N=\left\{\theta_{1}=0, \theta_{2}=0\right\}, N S=\left\{\theta_{1}=0, \theta_{2}=\pi\right\}, S N=\left\{\theta_{1}=\pi, \theta_{2}=0\right\}$,

\footnotetext{
${ }^{25}$ One may also set $\psi$ to have period $2 \pi$ yielding $T^{1,1} / \mathbb{Z}_{2}$ which is a also Sasaki-Einstein manifold. In fact, this is the horizon manifold of the complex cone over $\mathbb{F}_{0} \simeq \mathbb{C P}^{1} \times \mathbb{C P}^{1}$. Note that one must be careful to ensure that the Killing spinors are well-defined on making such identifications.
} 
$S S=\left\{\theta_{1}=\pi, \theta_{2}=\pi\right\}$ - these are all copies of the fibre circle over the corresponding point on the base $S^{2} \times S^{2}$. The outward pointing primitive normal vectors to the cone are computed to be

$$
v_{1}=[1,0,-1], \quad v_{2}=[0,1,-1], \quad v_{3}=[0,-1,0], \quad v_{4}=[-1,0,0] .
$$

Notice that these indeed form a good cone, as defined in the main text. Also notice that the vectors $\left\{v_{i}\right\}$ span $\mathbb{Z}^{3}$ over $\mathbb{Z}$. Lerman's theorem then states that the base of the metric cone is simply-connected, which is of course correct. Moreover, the fact that there are four facets means that $\pi_{2}\left(T^{1,1}\right) \cong \mathbb{Z}$, again correct.

We may now apply the Delzant theorem. The kernel is trivially calculated to be $(1,-1,-1,1)$. Thus the theorem gives a $U(1)$ gauged linear sigma model on $\mathbb{C}^{4}$ with charges $(1,-1,-1,1)$ - this is of course well-known to give the conifold. Turning on the FI parameter $t>0, t<0$ gives the two small resolutions of the conifold, related by the flop transition.

We now apply the $S L(3 ; \mathbb{Z})$ transformation

$$
\left(\begin{array}{ccc}
1 & 1 & 2 \\
0 & -1 & -1 \\
0 & 0 & -1
\end{array}\right)
$$

to the torus $\mathbb{T}^{3}$ of symmetries. The normal vectors now read

$$
v_{1}^{\prime}=[-1,1,1], \quad v_{2}^{\prime}=[-1,0,1], \quad v_{3}^{\prime}=[-1,1,0], \quad v_{4}^{\prime}=[-1,0,0] .
$$

These all lie in the plane at $e_{1}=-1$. Dropping this gives vectors in $\mathbb{R}^{2}$ :

$$
\mathcal{V}_{1}=[1,1], \quad \mathcal{V}_{2}=[0,1], \quad \mathcal{V}_{3}=[1,0], \quad \mathcal{V}_{4}=[0,0]
$$

The toric diagram may thus be embedded in the orbifold $\mathbb{C}^{3} / \mathbb{Z}_{2} \times \mathbb{Z}_{2}$ and is presented below.

We may also analyse the topology of the Kähler quotient directly, as in the main text. The $D$-term constraint reads

$$
\left|z_{1}\right|^{2}+\left|z_{2}\right|^{2}-\left|z_{3}\right|^{2}-\left|z_{4}\right|^{2}=t
$$

Setting $t=0$ one obtains a singular space - the conifold. Defining gauge invariant coordinates $u=z_{1} z_{3}, x=z_{1} z_{4}, y=z_{2} z_{3}, v=z_{2} z_{4}$ we have precisely one relation $u v=x y$ in $\mathbb{C}^{4}$, which is thus an equivalent definition of the conifold. 
Figure 4: Toric diagram of the conifold embedded in the orbifold $\mathbb{C}^{3} / \mathbb{Z}_{2} \times \mathbb{Z}_{2}$.

At $z_{3}=z_{4}=0$ we have a copy of $\mathbb{C P}^{1}=S^{2}$, of size $t$. On a patch in which $z_{2} \neq 0$ we may introduce a gauge invariant complex coordinate $z=z_{1} / z_{2}$. This patch covers a neighbourhood of the south pole at $z_{1}=0$. Similarly the coordinate $z^{\prime}=z_{2} / z_{1}$ covers a neighbourhood of the north pole at $z_{2}=0$. Over the intersection of the patches we have the relation $z^{\prime}=1 / z$, thus making the Riemann sphere. Let us now turn to the remaining coordinates. Consider the subspace in which $z_{2} \neq 0$ and introduce gauge invariant coordinates $x_{2}=z_{3} z_{2}, y_{2}=z_{4} z_{2}$. Thus, over the open set $U_{2}=\left\{z_{2} \neq 0, z_{3}=z_{4}=0\right\} \subset \mathbb{C P}^{1}$, our subspace looks like a trivial rank two bundle $\mathbb{C}^{2} \times U_{2}$. Similarly, over $U_{1}=\left\{z_{1} \neq 0, z_{3}=z_{4}=0\right\} \subset \mathbb{C P}^{1}$ we also have $\mathbb{C}^{2} \times U_{1}$, where the fibre is coordinatised by $x_{1}=z_{3} z_{1}, y_{1}=z_{4} z_{1}$. On the overlap $U_{1} \cap U_{2}$ we have the relation $x_{1}=x_{2}\left(z_{1} / z_{2}\right), y_{1}=y_{2}\left(z_{1} / z_{2}\right)$. By definition, this gluing gives the bundle $\mathcal{O}_{\mathbb{C P}^{1}}(-1) \oplus \mathcal{O}_{\mathbb{C P}^{1}}(-1)$, which is the resolved conifold.

The boundary, or horizon, of this manifold is an $S^{3}$ bundle over $\mathbb{C P}^{1}=S^{2}$, since $S^{3}$ is the boundary of $\mathbb{C}^{2}$. There are various ways of seeing the topology of the horizon. One way is to projectivise the original bundle. Recall that to projectivise a rank two complex vector bundle, with transition functions in $U(2)$, means that one replaces each $\mathbb{C}^{2}$ fibre with $\mathbb{C P}^{1}$, and glues the fibres together across overlaps using the induced transition functions, which lie in $U(2) / U(1)_{D} \cong S O(3)$. Here $S O(3)$ acts on the $\mathbb{C P}^{1}=S^{2}$ fibre in the usual way. Since the transition functions of $\mathcal{O}_{\mathbb{C P}^{1}}(-1) \oplus \mathcal{O}_{\mathbb{C P}^{1}}(-1)$ are diagonal, the projectivisation is just the product $\mathbb{C P}^{1} \times \mathbb{C P}^{1}$. The $U(1)$ factor we projected out has unit winding over the fibre $S^{2}$, since $S^{3} \rightarrow S^{2}$ is the Hopf map which has Chern number 1 . The winding is also 1 over the base since we began with the sum of two copies of $\mathcal{O}_{\mathbb{C P}^{1}}(-1)$. Thus we see explicitly the topology of $T^{1,1}$ as the horizon manifold. 


\section{References}

[1] J. M. Maldacena, "The large N limit of superconformal field theories and supergravity," Adv. Theor. Math. Phys. 2, 231 (1998) [Int. J. Theor. Phys. 38, 1113 (1999)] arXiv:hep-th/9711200

[2] A. Kehagias, "New type IIB vacua and their F-theory interpretation," Phys. Lett. B 435, 337 (1998) arXiv:hep-th/9805131.

[3] I. R. Klebanov and E. Witten, "Superconformal field theory on threebranes at a Calabi-Yau singularity," Nucl. Phys. B 536, 199 (1998) arXiv:hep-th/9807080.

[4] B. S. Acharya, J. M. Figueroa-O'Farrill, C. M. Hull and B. Spence, "Branes at conical singularities and holography," Adv. Theor. Math. Phys. 2 (1999) 1249 arXiv:hep-th/9808014.

[5] D. R. Morrison and M. R. Plesser, "Non-spherical horizons. I," Adv. Theor. Math. Phys. 3, 1 (1999) arXiv:hep-th/9810201.

[6] J. P. Gauntlett, D. Martelli, J. Sparks and D. Waldram, "Sasaki-Einstein metrics on $S^{2} \times S^{3}$," Adv. Theor. Math. Phys. 8, 711 (2004) arXiv:hep-th/0403002

[7] J. P. Gauntlett, D. Martelli, J. Sparks and D. Waldram, "Supersymmetric AdS 5 solutions of M-theory," Class. Quant. Grav. 214335 arXiv:hep-th/0402153.

[8] J. Cheeger, G. Tian, "On the cone structure at infinity of Ricci flat manifolds with Euclidean volume growth and quadratic curvature decay," Invent. Math. 118 (1994), no. 3, 493-571

[9] K. Intriligator and B. Wecht, "The exact superconformal R-symmetry maximizes a," Nucl. Phys. B 667, 183 (2003) arXiv:hep-th/0304128

[10] S. S. Gubser, "Einstein manifolds and conformal field theories," Phys. Rev. D 59 (1999) 025006 arXiv:hep-th/9807164

[11] B. Feng, A. Hanany and Y. H. He, "D-brane gauge theories from toric singularities and toric duality," Nucl. Phys. B 595, 165 (2001) arXiv:hep-th/0003085.

[12] C. P. Herzog and J. Walcher, "Dibaryons from exceptional collections," JHEP 0309, 060 (2003) arXiv:hep-th/0306298 
[13] C. P. Herzog, "Exceptional collections and del Pezzo gauge theories," JHEP 0404, 069 (2004) arXiv:hep-th/0310262.

[14] M. Bertolini, F. Bigazzi and A. L. Cotrone, "New checks and subtleties for AdS/CFT and a-maximization," JHEP 0412, 024 (2004) arXiv:hep-th/0411249.

[15] J. P. Gauntlett, D. Martelli, J. F. Sparks and D. Waldram, "A new infinite class of Sasaki-Einstein manifolds," Adv. Theor. Math. Phys. 8, 987 (2006) arXiv:hep-th/0403038.

[16] J. P. Gauntlett, D. Martelli, J. Sparks and D. Waldram, "Supersymmetric AdS backgrounds in string and M-theory," arXiv:hep-th/0411194.

[17] W. Chen, H. Lu, C. N. Pope, and J. F. Vazquez-Poritz, "A Note on EinsteinSasaki Metrics in $D \geq 7$," arXiv:hep-th/0411218.

[18] G. Tian, "On Kähler-Einstein metrics on certain Kähler manifolds with $c_{1}(M)>$ 0," Invent. Math. 89 (1987) 225-246.

[19] G. Tian and S.T. Yau, "On Kähler-Einstein metrics on complex surfaces with $C_{1}>0$," Commun. Math. Phys. 112 (1987) 175-203.

[20] E. Lerman, "Contact toric manifolds," J. Symplectic Geom. 1 (2003), no. 4, 785-828 math.SG/0107201.

[21] T. Delzant, "Hamiltoniens periodiques et images convexes de l'application moment," Bull. Soc. Math. France 116 (1988), 3, 315-339.

[22] E. Witten, "Phases of $\mathcal{N}=2$ theories in two dimensions," Nucl. Phys. B 403, 159 (1993) arXiv:hep-th/9301042.

[23] C. Beasley, B. R. Greene, C. I. Lazaroiu and M. R. Plesser, "D3-branes on partial resolutions of abelian quotient singularities of Calabi-Yau threefolds," Nucl. Phys. B 566, 599 (2000) arXiv:hep-th/9907186.

[24] E. Witten, "Baryons and branes in anti de Sitter space," JHEP 9807, 006 (1998) arXiv:hep-th/9805112.

[25] D. Berenstein, C.P. Herzog, I.R. Klebanov, "Baryon Spectra and AdS/CFT Correspondence," JHEP 0206 (2002) 047, arXiv:hep-th/0202150. 
[26] S. Smale, "On the structure of 5-manifolds," Ann. Math. 75 (1962), 38-46.

[27] Th. Friedrich and I. Kath, "Einstein manifolds of dimension five with small first eigenvalue of the Dirac operator," J. Differential Geom. 29 (1989) 263-279.

[28] Y. Matsushima, "Sur la structure du groupe d'homéomorphismes analytiques d'une certaine variété kaehlérienne," Nagoya Math. J. 11 (1957), 145-150.

[29] C. P. Boyer and K. Galicki, "New Einstein metrics in dimension five," J. Differential Geom. 57 (2001), no. 3, 443-463, arXiv:math.DG/0003174.

[30] C. P. Boyer, K. Galicki and M. Nakamaye, "On the Geometry of SasakianEinstein 5-Manifolds," Math. Ann. 325 (2003), no. 3, 485-524, arXiv:math.dg/0012047.

[31] C. P. Boyer, K. Galicki and M. Nakamaye, "Sasakian-Einstein structures on $9 \#\left(S^{2} \times S^{3}\right)$," Trans. Amer. Math. Soc. 354 (2002), no. 8, 2983-2996, arXiv:math.DG/0102181

[32] C. P. Boyer and K. Galicki, "New Einstein metrics on $8 \#\left(S^{2} \times S^{3}\right)$," Differential Geom. Appl. 19 (2003), no. 2, 245-251, arXiv:math.DG/0208202.

[33] M. Y. Wang and W. Ziller, "Einstein metrics on principal torus bundles," J. Diff. Geom. 31 (1990) 215

[34] C.P. Boyer, K. Galicki, "3-Sasakian Manifolds," Surveys Diff. Geom.7 (1999) 123-184, arXiv:hep-th/9810250.

[35] S. Falcao de Moraes and C. Tomei, "Moment maps on symplectic cones," Pacic J. Math. 181 (2) (1997), 357-375.

[36] M. F. Atiyah, "Convexity and commuting Hamiltonians," Bull. London Math. Soc. 14 (1982), 1-15.

[37] V. Guillemin and S. Sternberg, "Convexity properties of the moment mapping," Invent. Math. 67 (1982), 491-513.

[38] E. Lerman and S. Tolman, "Hamiltonian torus actions on symplectic orbifolds and toric varieties," da-ga/9511008. 
[39] C. P. Boyer and K. Galicki, "A Note on Toric Contact Geometry", arXiv:math.DG/9907043.

[40] E. Lerman, "Homotopy Groups of K-Contact Toric Manifolds," Trans. Amer. Math. Soc. 356 (2004), no. 10, 4075-4083 math.SG/0204064.

[41] D.N. Page, "A compact rotating gravitational instanton," Phys. Lett. 79B, No. 3 (1978) 235-238.

[42] B. Feng, S. Franco, A. Hanany and Y. H. He, "Symmetries of toric duality," JHEP 0212, 076 (2002) arXiv:hep-th/0205144.

[43] G. Dall'Agata, " $N=2$ conformal field theories from M2-branes at conifold singularities," Phys. Lett. B 460, 79 (1999) arXiv:hep-th/9904198.

[44] D. Fabbri, P. Fre', L. Gualtieri, C. Reina, A. Tomasiello, A. Zaffaroni and A. Zampa, "3D superconformal theories from Sasakian seven-manifolds: New nontrivial evidences for AdS(4)/CFT(3)," Nucl. Phys. B 577, 547 (2000) arXiv:hep-th/9907219.

[45] A. Ceresole, G. Dall'Agata, R. D'Auria and S. Ferrara, "M-theory on the Stiefel manifold and 3d conformal field theories," JHEP 0003 (2000) 011 arXiv:hep-th/9912107.

[46] K. Intriligator and B. Wecht, "Baryon charges in 4D superconformal field theories and their AdS duals," Commun. Math. Phys. 245, 407 (2004) arXiv:hep-th/0305046.

[47] P. Candelas and X. C. de la Ossa, "Comments On Conifolds," Nucl. Phys. B 342, 246 (1990). 